\title{
Levosimendan prevents tau pathology by inhibiting disulfide-linked tau oligomerization posing as a promising anti-tau therapeutics
}

Yun Kyung Kim ( $\nabla$ yunkyungkim@kist.re.kr)

Korea Institute of Science and Technology https://orcid.org/0000-0002-8682-120X

Sungsu Lim

Korea Institute of Science and Technology

Seulgi Shin

Korea Institute of Science and Technology

Ha Eun Lee

Korea Institute of Science and Technology

Ji Yeon Song

Korea Institute of Science and Technology

Yoonsik Sung

Korea Institute of Science and Technology

Gwan-Ho Lee

Korea Institute of Science and Technology

Byung-Yong Yu

Korea Institute of Science and Technology

Hira Aziz

Korea Institute of Science and Technology

Nataliia Lukianenko

Korea Institute of Science and Technology

Kyu Hyeon Kim

Korea Institute of Science and Technology

Hyeanjeong Jeong

Korea Institute of Science and Technology

Sang Min Lim

Korea Institute of Science and Technology

Jun-Seok Lee

Korea Institute of Science and Technology

Ae Nim Pae

Korea Institute of Science and Technology 
Article

Keywords: drug development, molecular medicine, tau pathology

Posted Date: December 30th, 2020

DOl: https://doi.org/10.21203/rs.3.rs-128169/v1

License: (c) (i) This work is licensed under a Creative Commons Attribution 4.0 International License. Read Full License 


\section{Abstract}

Tau oligomers play critical roles in tau pathology, responsible for neuronal cell death and transmitting the disease in the brain. Accordingly, preventing tau oligomerization becomes an important therapeutic strategy to treat tauopathies including Alzheimer's disease, however progress has been slow due to difficulties of detecting tau oligomers in cellular context. Toward tau-targeted drug discovery, our group have developed a tau-BiFC platform to monitor and quantify tau oligomerization. By using the tau-BiFC platform, we screened 1,018 compounds in FDA-approved \& Passed Phase I drug library, and identified levosimendan as a potent anti-tau agent inhibiting tau oligomerization. ${ }^{14} \mathrm{C}$-isotope labeling of levosimendan identified that levosimendan covalently bound to tau cysteines, directly inhibiting disulfidelinked tau oligomerization. In addition, levosimendan was able to disassemble tau oligomers into monomers, and rescuing neurons from aggregation states. In comparison, the well-known anti-tau agents, methylene blue (MB) and LMTM, failed to protect neurons from tau-mediated toxicity, generating high-molecular weight tau oligomers. The administration of levosimendan also suppressed tau pathology in the brain, preventing cognitive declines in Tau ${ }^{\mathrm{P} 301 \mathrm{~L}} \mathrm{BiFC}$ transgenic mice. Although careful validation is required, here we present the potential of levosimendan as a disease modifying therapy for tauopathies targeting tau oligomerization.

\section{Introduction}

Naturally, tau is an extremely soluble protein containing a number of positively charged lysine residues ${ }^{1}$. The lysine residues play a role for binding negatively charged microtubules ${ }^{2}$. Upon binding, tau stabilizes microtubule and promotes microtubule assembly, which is critical for axonal outgrowth ${ }^{3,4}$. Under pathological condition, the charge balance between tau and microtubule is disrupted, and tau dissociates from microtubules ${ }^{5}$. The dissociated tau become a susceptible substrate of cytosolic enzymes that modify the states of tau phosphorylation, acetylation, or proteolytic cleavage ${ }^{6,7}$. Chemically and structurally modified tau becomes aggregated and accumulated in neurons. Intraneuronal deposits of tau fibrils (PHFs and NFTs) are the pathological hallmark in a group of neurodegenerative diseases, called tauopathies. Tauopathies include not only Alzheimer's disease (AD), but also Fronto-Temporal Dementia (FTD), progressive supranuclear palsy, corticobasal degeneration, and chronic traumatic encephalopathy ${ }^{8,9}$. Due to the implication of tau in neurodegenerative disorders, tau has become an important therapeutic target ${ }^{10}$. However, progress has been slow due to the lack of understanding tau pathology.

Until recently, neurotoxic tau species were presumed to be the filamentous tau (PHFs and NFTs), accumulated in the brain of AD patients. However, mounting evidence suggests that soluble tau oligomer, rather than fibrils, is neurotoxic species responsible for neuronal cell death ${ }^{11,12}$. Tau oligomers are soluble forms of tau aggregates ranging from dimers to prefibrillar aggregates ${ }^{13}$. In $A D$ brains, tau oligomers are detected in the early stage of pathogenic cascades, and the level of tau oligomers correlated with synaptic dysfunction and neuronal cell loss, rather than the levels of NFTs ${ }^{14-16}$. Evidence also suggested 
that tau oligomers are transmittable between neurons, spreading the disease in the brain ${ }^{17,18}$. Due to its relatively small size, tau oligomers are able to penetrate into cells and initiate nascent tau aggregation, providing structural seeds for aggregation ${ }^{19}$. The formation of insoluble filament (PHFs and NFTs) might be a survival mechanism for neurons to quarantine toxic tau species from cytosol ${ }^{20}$. Accordingly, prevention of tau oligomerization or elimination of oligomers became an important therapeutic strategy for $A D$ drug development.

The most studied tau aggregation inhibitor is methylene blue (MB), a blue dye, which has been widely used in various industrial and research settings. In 2008, Wischik et al. firstly reported the anti-tau activity of $\mathrm{MB}^{21}$. In their study, MB disrupted the stability of NFTs isolated from AD patients. In 2013, Zweckstetter et al. reported the inhibitory mechanism of MB on tau aggregation. In their study, MB inhibits tau aggregation through the oxidation of tau cysteine residues, converting tau into aggregationincompetent monomer ${ }^{22}$. However, there are also conflicting data showing that MB increased tau dimers through the same cysteine-oxidation mechanism ${ }^{23}$. Full length tau contains two cysteine residues (C291 and C322) in the microtubule-binding domain. Intra-molecular disulfide bond formation generates a compact monomer, resistant to tau aggregation, and inter-molecular disulfide bonds facilitate the aggregation cascade by generating structurally stable tau oligomers ${ }^{24}$. In 2019, Takashima et al. reported that MB inhibited the formation of tau fibrils, but not tau oligomers ${ }^{25}$. While the mechanism of action on tau pathology is still unclear, the reduced form of MB, LMTM (also known as TauRx0237 or LMT-X), has completed Phase III clinical trials for the treatment of Alzheimer's disease. In first Phase III trial, LMTM failed to ameliorate cognitive decline in patients with mild to moderate Alzheimer's disease ${ }^{26,27}$. Although failed in AD treatment, LMTM is still on clinical trials for the treatment of FTDs.

Tau aggregation is a complicated multi-step process, which is controlled by a number of cellular enzymes. Therefore, in many cases, tau aggregation inhibitors which show strong inhibitory effects in in vitro tau aggregation assays, are not effective in cellular system. Therefore, the action-mechanism of tautargeted drugs should be evaluated in cellular system, not in test tubes using purified tau. In intracellular space, diverse tau modifications occur simultaneously, changing its physiological properties; tau phosphorylation reducing its microtubule binding affinity ${ }^{28,29}$, tau acetylation preventing ubiquitinmediated degradation ${ }^{30}$, and dityrosine or disulfide-bond formation promoting tau aggregation by generating covalently linked tau oligomers ${ }^{24,31}$. In this regard, our group has developed tau-BiFC platform to monitor tau oligomerization in living cells and in the brain of mice ${ }^{32,33}$. In tau-BiFC system, nonfluorescent $\mathrm{N}$ - and $\mathrm{C}$-terminal compartments of Venus protein are fused to tau and Venus fluorescence turns on only when tau assembles together (Fig. 1a). By using tau-BiFC cell model, we have characterized prion-like tau oligomers ${ }^{19}$, and investigated pathological tau modification ${ }^{34-36}$.

In this study, by using tau-BiFC platform, we have screened FDA-approved \& Passed Phase I Drug Library, and identified levosimendan as a new anti-tau agent.

\section{Results}




\section{FDA-approved \& Passed Phase I Drug Library screening identifies levosimendan as a new anti-tau oligomerization agent}

To identify a new drug candidate inhibiting tau aggregation, we screened 1,018 compounds in the FDAapproved \& Passed Phase I Drug Library on tau-BiFC cells. For tau-BiFC screening, tau-BiFC cells were plated on 384-well and cells were co-treated with each library drug with Forskolin (FK), which induces tau hyperphosphorylation through the activation of Protein kinase $A^{37}$ (Fig. 1a). MB and LMTM (3 mM) were used as a positive control. After 46 hours, FK treatment induced tau aggregation by showing $3.0 \pm 0.4$-fold increased BiFC fluorescence intensity (Fig. 1a-d). FK treatment also induced cell cytotoxicity by showing $26.6 \pm 0.2 \%$ decreased number of nuclei (Fig. 1b, c, and e). Among tested, only one drug, levosimendan suppressed FK-induced tau aggregation almost completely to the level of basal without showing any cytotoxic effects $\left(E C_{50}=1.9 \pm 0.1 \mathrm{mM}, \mathrm{GI}_{50}=124.0 \pm 0.3 \mathrm{mM}\right.$ ) (Fig. $1 \mathrm{f}$ and Supplementary Fig. 1). More interestingly, FK-induced cell toxicity was rescued to the level of basal by the treatment of levosimendan. In comparison, MB and LMTM decreased cell viability seriously near at the effective concentration (MB; $\mathrm{EC}_{50}=2.8 \pm 0.2 \mathrm{mM}, \mathrm{GI}_{50}=6.2 \pm 0.4 \mathrm{mM}$, and $\mathrm{LMTM} \mathrm{EC}_{50}=2.2 \pm 0.2 \mathrm{mM}, \mathrm{Gl}_{50}=6.4 \pm 0.3 \mathrm{mM}$ ) (Fig. $1 \mathrm{f}$ and Supplementary Fig. 1).

Tau-BiFC cell screening identified that levosimendan is an effective drug in preventing FK-induced tau aggregation and also FK-induced cellular toxicity. However, FK activates not only tau kinases, but also other cellular processes activated with the increased level of CAMP ${ }^{38}$. The inhibitory effect of levosimendan could be the result of regulating the upstream cAMP pathway, rather than tau pathology. To scrutinize the drug effect on tau pathology, tauK $18^{\mathrm{P} 301 \mathrm{~L}}$ was used to activate tau aggregation. TauK18 fragment containing $\mathrm{P} 301 \mathrm{~L}$ mutation, tauK $18^{\mathrm{P} 301 \mathrm{~L}}$, activates tau aggregation as a prion-like seed ${ }^{19}$.

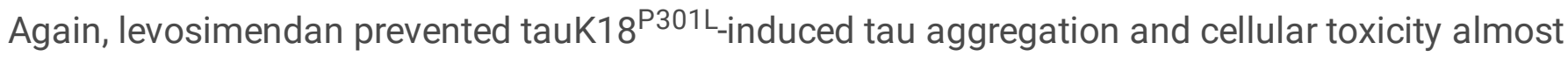
completely $\left(\mathrm{EC}_{50}=2.2 \pm 0.1 \mathrm{mM}, \mathrm{Gl}_{50}=101.3 \pm 0.4 \mathrm{mM}\right.$ ) (Fig. $1 \mathrm{~g}$ and Supplementary Fig. 2). MB and LMTM showed serious cell toxicity near at effective concentration (MB; $\mathrm{EC}_{50}=3.1 \pm 0.3 \mathrm{mM}, \mathrm{Gl}_{50}=7.1 \pm 0.3 \mathrm{mM}$, and $\mathrm{LMTM} ; \mathrm{EC}_{50}=2.7 \pm 0.2 \mathrm{mM}, \mathrm{GI}_{50}=6.6 \pm 0.3 \mathrm{mM}$ ) (Fig. $1 \mathrm{~g}$ and Supplementary Fig. 2).

Next, flow cytometry analysis was conducted to investigate drug effect on single-cell level. Since MB and LMTM caused substantial cell death at effective concentration, flow cytometry analysis was necessary to validate the drug effect on the remaining intact cells. For flow cytometry analysis, tau-BiFC cells were treated with the most effective concentration of each drugs. Upon the treatment of tauK $18^{\mathrm{P} 301 \mathrm{~L}}, 65.4 \%$ of total cells were selected as an intact cell population on forward and side scatter pulse area (FSC-A and SSC-A) (Fig. 1h). In case of MB and LMTM (5 mM), 13.7\% and $14.5 \%$ of total cells were selected as an intact cell population. In comparison, $69.0 \%$ of levosimendan $(15 \mathrm{mM})$ treated cells were selected as intact cells. The selected populations were analyzed for BiFC fluorescence intensity. Tau-BiFC cells are mixture of BiFC-null cells and BiFC-responding cells (Fig. 1i). In the basal condition, BiFC-responding cells showed low and widespread BiFC fluorescence profile (BiFC OFF cells; Median $=332 \pm 110$ ). Upon tauK18 ${ }^{\mathrm{P} 301 \mathrm{~L}}$ activation, the fluorescence intensities of BiFC-responding cells increased significantly generating a distinctive population peak $\left(\mathrm{BiFC}^{\mathrm{ON}}\right.$ cells; Median $\left.=717 \pm 62\right)$, while BiFC-null cells still 
remained. The fluorescence intensity of $\mathrm{BiFC}^{\mathrm{ON}}$ cells dropped significantly by the treatment of levosimendan (Levo; Median = 249 \pm 59 ), proving the effectiveness of levosimendan in inhibiting cellular tau aggregation. In comparison, MB or LMTM reduced tau-BiFC intensity only slightly (MB; Median = $648 \pm 49$, and LMTM; Median $=596 \pm 57$ ). High magnification images show the representative BiFC fluorescence images of each group (Fig. 1j). Upon the treatment of tauK18 $8^{\mathrm{P} 301 \mathrm{~L}}$, intracellular tau aggregation increased significantly showing thread-like phenotype, and the increased BiFC fluorescence was suppressed almost completely by the treatment of levosimendan. As shown in flow cytometry analysis, MB and LMTM was not effective in reducing intracellular tau aggregation.

\section{Levosimendan inhibits intracellular disulfide-linked tau oligomerization while MB and LMTM increase disulfide-linked tau oligomers.}

Next, immunoblot analysis was followed to evaluate tau phosphorylation and total tau levels in tau-BiFC cells. To investigate dose-dependency, tau-BiFC cells were treated with increasing concentrations of each drug in the presence of tauK18 $8^{\text {P301L }}$ (MB and LMTM; 0.5, 1.5, $5 \mathrm{mM}$, and levosimendan; 5, 15, $45 \mathrm{mM}$ ). Upon the activation by tauK $18^{\mathrm{P} 301 \mathrm{~L}}$, the levels of tau phosphorylation and total tau were increased by $1.9 \pm 0.1$-fold at T205, 1.6 \pm 0.1 -fold at S199, $1.4 \pm 0.1$-fold at S396 and 2.1 \pm 0.1 -fold, compared to that of basal (Fig. 2a-c and Supplementary Fig. 3). The increased tau phosphorylation was reduced by the treatment of levosimendan, and at the highest concentration $(45 \mathrm{mM})$, the levels of tau phosphorylation was decreased almost to the level of basal, showing $0.9 \pm 0.1$-fold at T205, 1.0 \pm 0.1 -fold at S199 and $0.9 \pm 0.1$-fold at S396 (Fig. 2a, b and Supplementary Fig. 3). Upon the treatment of MB and LMTM, the level of tau monomers was dose-dependently decreased, but instead, high-molecular weight oligomers were observed. In case of MB (5 mM), the level of pT205-, pS199-, or pS396-positive monomers was decreased to $0.1 \pm 0.9-, 0.2 \pm 0.1$-, or $0.2 \pm 0.1$-fold and the level of corresponding oligomers was increased to $0.7 \pm 0.3-, 0.4 \pm 0.1-, 0.5 \pm 0.2$-fold, respectively, compared to that of basal. In case of LMTM ( $5 \mathrm{mM})$, the level of pT205-, pS199-, or pS396-positive monomers was decreased to $0.2 \pm 0.9-, 0.3 \pm 0.0-$, or $0.2 \pm 0.1$-fold and the level of oligomers was increased to $0.6 \pm 0.2-, 0.4 \pm 0.1$-, or $0.4 \pm 0.1-f o l d$, respectively. This result indicates that MB and LMTM increase unbreakable tau oligomers, which are resistant to SDS- and also bmercaptoethanol. Also, the size of oligomers was increased dose-dependently in MB and LMTM treated cell lysates.

Upon the treatment of tauK $18^{\mathrm{P} 301 \mathrm{~L}}$, the amount of total tau protein was increased to $2.0 \pm 0.1$-fold

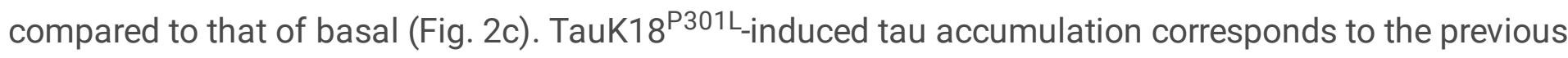
report showing that tau is metabolized at much slower rates when pathologically activated ${ }^{39}$.

Levosimendan treatment effectively suppressed tauK18 ${ }^{\mathrm{P} 301}$-induced tau accumulation in tau-BiFC cells, keeping total tau level at 1.0 \pm 0.2 -fold, which is comparable to the level of basal. Next, we investigated the formation of disulfide-dependent tau oligomers on non-reducing SDS-PAGE gels. Substantial amount of disulfide-linked tau oligomers were detected even at basal condition and the level of disulfide-linked tau oligomers increased to $2.4 \pm 0.4$-fold by the treatment of tauK18 ${ }^{\mathrm{P} 301 \mathrm{~L}}$ (Fig. $2 \mathrm{~d}$ ). Levosimendan treatment decreased the amount of disulfide-linked tau oligomers, comparable to the basal level $(0.8 \pm 0.2$-fold at 45 
$\mathrm{mM}$ ). In contrast, MB and LMTM increased the level of disulfide-linked tau oligomerization intensely (MB; 1.6 \pm 0.3-fold and LMTM; $1.9 \pm 0.3$-fold at $5 \mathrm{mM}$ ), presenting highly enriched bands above $245 \mathrm{kDa}$ (Fig. 2d). Non-reducing gel analysis indicates that levosimendan is effective in inhibiting tau-BiFC responses through the suppression of disulfide-linked tau oligomerization. In comparison, MB and LMTM did not inhibit tau-BiFC responses and promoted the formation of structurally stable tau oligomers that are SDSand reducing agent resistant. However, careful validation was necessary since it was possible that $\mathrm{MB}$ and LMTM might act on BiFC complementation process instead of tau oligomerization.

Next, we evaluated the drug effect on tau oligomerization in primary neuron culture. Primary hippocampal neurons were isolated from day18 rat embryos. After 10 days of in vitro culture, the hippocampal neurons were treated with MB, LMTM $(3 \mathrm{mM})$ or levosimendan $(10 \mathrm{mM})$ in the presence of tauK18 $8^{\mathrm{P} 301 \mathrm{~L}}$. In case of MB and LMTM, severe neuronal cell death was observed at higher concentrations over $10 \mathrm{mM}$, similar to those toxicity on tau-BiFC cells (data not shown). The neuronal cell lysates were subjected for immunoblot analysis in both reducing- and non-reducing- condition. Upon the treatment of tauK18 $8^{\mathrm{P} 301 \mathrm{~L}}$, tau phosphorylation increased 2.0 \pm 0.2 -fold at S396 and 1.7 \pm 0.1 -fold at S199, indicating activated tau pathology in primary neuron (Fig. 2e, f). Again, levosimendan treatment suppressed tauK18 ${ }^{\mathrm{P} 301 \mathrm{~L}-\text {-induced }}$ tau phosphorylation comparable to the basal level, showing $0.9 \pm 0.2$-fold at S396 and $0.9 \pm 0.1$-fold at S199. Also, in the primary neurons, the treatment of MB and LMTM increased the level of high-molecular weight tau oligomers resistant to SDS- and b-mercaptoethanol, while decreasing the level of monomers (Fig. 2e, f). Upon the treatment of tauK18 ${ }^{\mathrm{P} 301 \mathrm{~L}}$, the level of total tau increased to $1.5 \pm 0.1$-fold compared to that of basal, indicating the accumulation of pathological tau species in neurons (Fig. $2 \mathrm{~g}$ ). Levosimendan treatment also suppressed tau accumulation in neurons, maintaining total tau level at $1.0 \pm 0.1$-fold comparable to that of basal.

Non-reducing SDS-PAGE analysis also indicated that, substantial amount of tau exists as disulfide-linked tau oligomers in primary neurons (Fig. 2h). In basal condition, the level of disulfide-linked oligomers was $2.4 \pm 0.7$-fold, compared to the level of monomer. Upon tauK $18^{\mathrm{P} 301 \mathrm{~L}}$ activation, the amount of disulfidelinked tau oligomers was increased by $1.8 \pm 0.3$-fold compared to that of basal. Levosimendan efficiently suppressed the formation of disulfide-linked oligomers, decreasing the level of oligomers to $0.5 \pm 0.1$-fold, which is even lower than that of basal. In case of MB and LMTM, the level of oligomers was increased to 2.3 \pm 0.5 -and 2.4 \pm 0 .3-fold, respectively, and monomers were almost disappeared (Fig. $2 \mathrm{~h}$ ). Our results clearly indicated that while MB and LMTM increases the formation of tau oligomers in primary neurons, levosimendan prevented tau phosphorylation, inhibiting disulfide-linked tau oligomerization.

Non-reducing SDS-PAGE analysis indicated that significant amount of tau exists as disulfide-linked oligomers upon the activation of tau pathology. Full-length human tau has two cysteine residues (C291 and C322) that can form intra- and inter-molecular disulfide bonds ${ }^{40}$. While intramolecular disulfide bonds lead to the formation of compact monomers that cannot form extended structure, intermolecular disulfide-bonds produce covalently linked oligomers. The disulfide-linked oligomers serve as "nuclei" for further tau aggregation ${ }^{24}$ (Fig. 2i). Tau aggregation may occur in the absence of disulfide-bond 
formation, but disulfide-linked tau oligomers could facilitate tau aggregation, serving as a structural seed for tau aggregation. Our result indicates that the treatment of MB and LMTM increased the level of disulfide-linked tau oligomers and levosimendan prevented the formation of disulfide-linked tau oligomers.

Next, we evaluated the effects of tau oligomers on neuronal integrity. At DIV10, hippocampal neurons were treated with MB, LMTM (3 mM) or levosimendan $(10 \mathrm{mM})$ followed by tauK18 ${ }^{\mathrm{P} 301 \mathrm{~L}}$. After $48 \mathrm{hrs}$ of incubation, neurons were stained with NeuO, a neuron specific fluorescence dye ${ }^{41}$ (Fig. 2j). Then, fluorescence images were acquired and analyzed to evaluate the length of neurites (Fig. 2k).

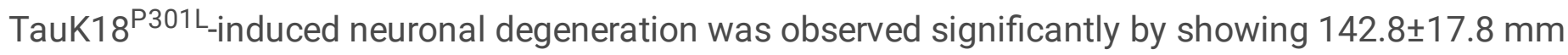
shortened neurites compared to that of basal $(p<0.0001)$. Levosimendan inhibited tauK18 $8^{\mathrm{P} 301 \mathrm{~L}}$-induced neuronal degeneration by recovering neurite length to $346.8 \pm 20.7 \mathrm{~mm}$. In contrast, MB did not show significant effect on neuronal integrity and LMTM protected neuronal degeneration slightly by showing $151.1 \pm 24.4 \mathrm{~mm}$ neurite length. This result clearly indicates that levosimendan prevented tauK $18^{\mathrm{P} 301 \mathrm{~L}}$ induced toxicity not only in tau-BiFC cells, but also in primary neurons, via inhibiting the formation of disulfide-dependent tau oligomers.

\section{Levosimendan not only prevents tau oligomerization, but also disaggregates filamentous tau.}

Next, we validated the direct effect of each drug on tau oligomerization and tau fibrilization in in vitro conditions using purified tauK18 fragment. For tau oligomerization analysis, tau pre-aggregates were incubated with each drug at various concentrations $(10,30$, and $100 \mathrm{mM})$ for 5 hours. Then, tau oligomers in the mixtures were separated into reducing and non-reducing SDS-PAGE (Fig. 3a, b). In DMSO-treated control, $50 \pm 1.1 \%$ of total tau exists as disulfide-linked oligomers, which were formed spontaneously in phosphate-buffered saline ( $\mathrm{pH} 7.4)$. Upon the treatment of levosimendan, the levels of disulfide-linked tau oligomers was decreased to $15.0 \pm 6.0 \%$ at $10 \mathrm{mM}, 15.7 \pm 7.8 \%$ at $30 \mathrm{mM}$, and $12.3 \pm 15.0 \%$ at $100 \mathrm{mM}$, increasing the level of monomers. This result indicates that levosimendan directly inhibits disulfide-linked tau oligomerization. In case of MB and LMTM, at $100 \mathrm{mM}$ concentration, the level of disulfide-linked tau oligomers was increased to $77.6 \pm 2.0 \%$ and $79.2 \pm 0.8 \%$, respectively. MBand LMTM-induced tau oligomers are not easily dissociable into monomers even in reducing condition, showing noticeable bands of dimers and trimers, resistant to SDS- \& b-mercaptoethanol on a reducing SDS-PAGE gel (Fig. 3b). In vitro tau oligomerization assay indicates that levosimendan prevents disulfidelinked tau oligomerization by direct interaction with tau protein, while MB and LMTM increase disulfidelinked tau oligomerization.

Then, we investigated the inhibitory effect on tau aggregation. For tau aggregation assay, tau aggregation was induced by the treatment of heparin to purified tauK18 in the presence of each drug at various concentrations for 5 days. The formation of $b$-sheet aggregates was evaluated with Thioflavin $S$ (ThS) assay. All drugs inhibited the formation of ThS-positive aggregates at micromolar concentration (MB; $\mathrm{EC}_{50}=3.1 \pm 0.1 \mathrm{mM}, \mathrm{LMTM} \mathrm{EC}_{50}=1.9 \pm 0.2 \mathrm{mM}$, and levosimendan; $\mathrm{EC}_{50}=2.6 \pm 0.1 \mathrm{mM}$ ) (Fig. 3c). Then, the structures of tau aggregates were evaluated under transmission electron microscopy (TEM) (Fig. 3d). 
TEM image of DMSO control shows the formation of long and straight filaments of tau. In comparison, tau filaments are not observed in levosimendan-treated mixture, indicating its effectiveness in inhibiting tau aggregation. In case of MB and LMTM, a few thread-like filaments were observed. We also investigated the activity of drugs on reversing tau aggregation. For disaggregation assay, tau aggregates were treated with each drug at various concentrations for 4 days. ThS assay indicated that all three drugs were effective in reducing ThS-positive tau aggregates $\left(M B ; E_{50}=3.8 \pm 0.1 \mathrm{mM}, \mathrm{LMTM} \mathrm{EC}_{50}=3.4 \pm 0.2 \mathrm{mM}\right.$, and levosimendan; $\mathrm{EC}_{50}=3.7 \pm 0.2 \mathrm{mM}$ ) (Fig. 3e). However, ThS results of $\mathrm{MB}$ and LMTM conflicted with TEM images showing thick bundles of tau filaments in MB- and LMTM-treated mixture (Fig. 3f). The result corresponds with a previous study showing, upon treated to tau filaments, MB did not reduced the tau filaments shown under electron microscope ${ }^{42}$. Our result implies that the treatment of MB or LMTM prevents the interaction between ThS and tau filaments, generating ThS-negative tau filaments. In case of levosimendan, ThS response correlated with high-resolution TEM image, which shows a bunch of short tau fragments. The lengths of tau filaments are ranging from $8.8 \mathrm{~nm}$ to $139.3 \mathrm{~nm}(\mathrm{n}=403)$. The in vitro tau aggregation and disaggregation assay show the effectiveness of levosimendan as an anti-tau oligomerization agent, which not only inhibits tau aggregation, but also disassembles tau filaments.

To further confirm the effectiveness of levosimendan on tau disassembly, levosimendan was treated at diverse time-points of tau aggregation processes in vitro and in tau-BiFC cells. For in vitro assay, tauK18 aggregation was induced by heparin, and the level of tau aggregation was monitored with ThS over 5 days. Levosimendan was treated to an aliquot of the aggregation mixture at $0,25,44$, and 75 hours after heparin activation (Fig. 3g). ThS-response curve shows that tau aggregation followed nucleationelongation mechanism. Levosimendan treatment, no matter when it was treated, decreased ThS-positive tau aggregates (Fig. 3h). For tau-BiFC assay, tau pathology was activated by the treatment of tauK18 ${ }^{\mathrm{P} 301 \mathrm{~L}}$, and tau-BiFC fluorescence intensities were monitored over three days. Tau-BiFC cells were treated with levosimendan at 1, 12, 24, and 36 hours after tauK18 ${ }^{\mathrm{P} 301 \mathrm{~L}}$ activation (Fig. 3i). Again, levosimendan treatment, no matter when it was treated, decreased tau-BiFC fluorescence responses, indicating the effectiveness as an anti-tau oligomerization agent (Fig. 3j and Supplementary Fig. 3).

\section{Levosimendan inhibits disulfide-crosslinked tau oligomerization in vitro}

Next we investigated the molecular mechanism of levosimendan in inhibiting oligomerization. We hypothesized that levosimendan inhibits tau-disulfide bond formation by blocking tau cysteine residues, since its nitrile group is able to form a thioimidate bond with cysteine. To verify its covalent modification to tau, one of two nitrile groups of levosimendan was labelled with ${ }^{14} \mathrm{C}$-radioisotope (Fig. $4 \mathrm{a}$ and Supplementary materials). ${ }^{14} \mathrm{C}$-levosimendan was treated to tau pre-aggregates that contains disulfidelinked tau oligomers for $2 \mathrm{hrs}$. Then, disulfide-linked tau oligomers were separated on a non-reducing SDS-PAGE gel (Fig. 4b, c). ${ }^{14} \mathrm{C}$-radiography showed tau monomers and dimers labelled with ${ }^{14} \mathrm{C}$ levosimendan, indicating its covalent attachment to tau (Fig. 4c). Then, the relative intensities of Coomassie blue-stained bands of monomer, dimer, trimer, and tetramer were compared (Fig. 4c, d). Corresponding to Figure $3 \mathrm{e}$, the treatment of ${ }^{14} \mathrm{C}$-levosimendan disassembled disulfide-linked tau 
oligomers, increasing the level of monomers as levosimendan did (Fig. 4c, d). In addition, high-resolution TEM images were acquired to validate the size of tau oligomers in DMSO-treated control and levosimendan-treated mixture. Heterogeneous tau particles with diameters of $13.7 \pm 5.2 \mathrm{~nm}$ were observed in DMSO-treated control $(n=60)(F i g .4 e, f)$. The diameters of tauK18 oligomers matches with a previous report showing that tauK18 formed spherical tau oligomers in size with diameters of $10 \sim 20 \mathrm{~nm}^{43}$. In comparison, comparably small tau particles with diameters of $8.8 \pm 2.0 \mathrm{~nm}$ were observed in levosimendan treated mixture $(n=69)$, supporting the disassembly of tau oligomers by levosimendan. To validate levosimendan-binding to tau cysteine residues, each repeat domain of tauK18 was synthesized and incubated with levosimendan (Fig. 4g). MALDI-mass spectrometry indicated that levosimendan covalently attached to R2 and R3 domains containing a cysteine residue.

Next, we confirmed the importance of the nitrile moiety of levosimendan in inhibiting tau aggregation by using its metabolites. In human, 5\% of levosimendan is metabolized to OR-1855 and OR-1896 in which the nitrile moiety is absent ${ }^{44}$ (Fig. 4h). In particular, OR-1896 is a pharmacologically active metabolite of levosimendan, acting as a powerful inodilator ${ }^{45}$. The metabolite's effects on tau aggregation were evaluated in vitro and in tau-BiFC cells. For in vitro assay, tau aggregation was induced by heparin in the presence of each metabolite for 5 days. ThS responses indicated that OR-1855 and OR-1896 did not inhibit tau aggregation in vitro, while levosimendan showed strong anti-tau aggregation activity (Fig. 4i). For tau-BiFC assay, tau-BiFC cells were treated with each metabolite for $48 \mathrm{hrs}$ in the presence of tauK18 ${ }^{\mathrm{P} 301 \mathrm{~L}}$. Again, even at $100 \mathrm{mM}$ concentration, OR-1855 and OR-1896 did not inhibit the tau-BiFC fluorescence increase (Fig. 4j). This result indicated that the nitrile group is critical in inhibiting tau aggregation through the modification of tau cysteine resides. Interestingly, OR-1896, which is a pharmacologically active metabolite acting as an inodilator, did not show any effect on tau. This result strongly suggests that the anti-tau activity is a new mode of action of levosimendan, distinguished from its known action as an inodilator.

\section{Levosimendan rescues cognitive deficit and tau pathology in Tau ${ }^{\mathrm{P} 301 \mathrm{~L}-\mathrm{BiFC}}$ mice.}

Next, we evaluated in vivo efficacy of levosimendan in Tau ${ }^{\text {P301L }}$-BiFC mice ${ }^{33}$. Tau ${ }^{\text {P301L }}$-BiFC mouse model expresses human mutant (P301L) tau labeled by BiFC compartments (Fig. 5a). In the brain of Tau ${ }^{\text {P301L }}$ $\mathrm{BiFC}$ mouse, tau oligomerization occurs from 3 months and neuronal degeneration occurs from 9 months, leading to cognitive deficits at 12 months of age. To evaluate the therapeutic effect of levosimendan in preventing neuronal degeneration and cognitive impairment, Tau ${ }^{\mathrm{P} 301 \mathrm{~L}-\mathrm{BiFC} \text { mice were }}$ received intraperitoneal administration of levosimendan or LMTM $(5 \mathrm{mg} / \mathrm{kg}$, three times per week) from 9 months to 12 months (Fig. 5a). After 4 month of drug administration, mice were subjected to behavioral tests to monitor cognitive function; novel objective test (NOR), Y-maze test, and passive avoidance test. For comparison, age-matched wild-type (WT) littermates were also subjected to the tests. In the novel objective recognition test, cognitive abilities were determined by the recognition index (RI) for a novel object. Vehicle-treated transgenic (TG) mice showed a significant decrease in the recognition index, with a RI value of $0.49(p<0.0001$, compare to WT), while WT mice exhibited 0.74 of a RI value for a novel 
object. Levosimendan-treatment significantly improved recognition memory performance with a RI value of $0.69(p<0.0001)$. LMTM-treatment also attenuated the recognition deficit of TG mice by showing a RI value of $0.59(p<0.01)$ (Fig. 5b). In Y-maze test, cognitive ability was determined by the percent alternation. Each levosimendan- and LMTM- treated group showed significant increase in the alternation (70.4 $\pm 4.2 \%, p<0.05$ and $68.9 \pm 3.5 \%, p<0.05$ compared to vehicle-treated, respectively), compared with the vehicle-treated group $(52.8 \pm 2.6 \%, p<0.01$ compared to WT) (Fig. $5 \mathrm{c}$ ). Further, passive avoidance test was followed to assess emotion-associated learning ability. Learning abilities were determined with the latency of entering the dark compartment, where the mice received an electrical shock a day before. Most mice in the vehicle-treated group entered the dark chamber without hesitation $(p<0.05)$, indicating the impairment of fear memory. Levosimendan-treated mice exhibited improved memory showing delayed latency or not entering to the chamber compared with the vehicle-treated TG controls $(p<0.05)$ (Fig. $5 \mathrm{~d}$ ). Interestingly, the LMTM-treated group, did not show significant improvement similar to vehicle-treated TG mice. All these results indicate that administration of levosimendan ameliorates tauopathy-induced memory deficits of aged, symptomatic tau TG mice.

Next, brains were extracted from mice, and brain tissue sections were prepared to evaluate tau pathology. Tau-BiFC fluorescence images indicate the level of tau assembly including tau oligomers and aggregates and AT8-immunostain indicates the level of tau phosphorylation associated with late-state of tau aggregation. Tau-BiFC intensities decreased both in LMTM and levosimendan-treated groups (Fig. 5e, g). In levosimendan treated group, tau-BiFC intensities decreased to $0.6 \pm 01$-fold in cortex and $0.4 \pm 0.2$-fold in hippocampus, compared to those of vehicle-treated group. In LMTM-treated group, tau-BiFC intensities decreased to $0.7 \pm 0.2$-fold in cortex and $0.6 \pm 0.1$-fold in hippocampus. AT8-immunoreactivity also decreased in both LMTM and levosimendan treated groups (Fig. $5 f, g$ ). In levosimendan-treated group, AT8-immunoreactivity decreased to $0.4 \pm 0.1$-fold in cortex and $0.4 \pm 0.2$-fold in hippocampus, compared to those of vehicle-treated group. In LMTM-treated group, AT8-immunoreactivity decreased to $0.6 \pm 0.2$-fold in cortex and 0.5 \pm 0.2 -fold in hippocampus.

Next, tau immunoblot assay was followed to evaluate drug effects on tau oligomerization and aggregation, using RIPA-soluble and RIPA-insoluble brain lysates. On tau immunoblots, mouse tau is indicated with black arrow ( $50 \mathrm{kDa})$ and human tau-BiFC compartments are indicated with two green arrows (76 and $85 \mathrm{kDa}$ ). In RIPA soluble brain lysates, tau phosphorylation levels were significantly decreased in LMTM- and levosimendan-treated groups (Fig. 5h). In levosimendan-treated group, the levels of tau phosphorylation decreased $0.5 \pm 0.0$-fold at S199 and $0.5 \pm 0.1$-fold at S396, compared to that of vehicle-treated group. In LMTM-treated group, the levels of tau phosphorylation decreased $0.6 \pm 0.1$-fold at S199 and 0.8 \pm 0.2 -fold at S396. Different from the previous results showing the increased level of SDSand b-mercaptoethanol resistant tau oligomers, LMTM-treated group did not show any high-molecular weight oligomers in the brain lysates (Fig. 5h). Also, the level of total tau decreased slightly in levosimendan treated group, although the decrease was not statically significant. Corresponding to AT8immunofluorescence images in Figure $5 f$, the amount of insoluble tau decreased $0.5 \pm 0.2$-fold in levosimendan-treated mice (Fig. 5i). In case of LMTM, the amount of insoluble tau decreased $0.7 \pm 0.1$ fold. Collectively, our results indicated that levosimendan is a new drug candidate targeting tau 


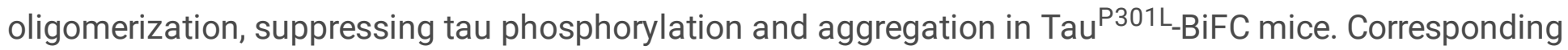
to AT8-immunofluorescence images in Figure $5 f$, the amount of insoluble tau decreased $0.5 \pm 0.2$-fold in levosimendan-treated mice (Fig. $5 \mathrm{i}$ ). In case of LMTM, the amount of insoluble tau decreased $0.7 \pm 0.1$ fold.

Next, the level of disulfide-linked tau oligomers was evaluated by non-reducing SDS-PAGE analysis. Similar to the result of primary neuron cultures, disulfide-linked tau oligomers were observed in the brain

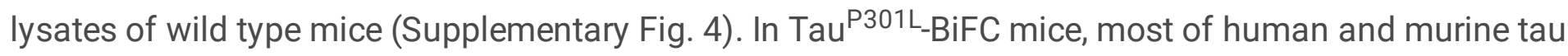
exists as the form of disulfide-linked oligomers (> $245 \mathrm{kDa}$ ) (Fig. 5j and Supplementary Fig. 4). Levosimendan-treatment significantly decreased the high-molecular weight oligomers and increased soluble monomeric tau level. This result is consistent with previous data showing that levosimendan inhibits the formation of disulfide-cross linkage between tau. In contrast, LMTM-treatment did not increase nor decrease the level of disulfide-linked tau oligomers in the brain (Fig. 5j). The LMTM effect in the animal was contrasted with the effect when LMTM interacts with tau directly in test tubes. This result implies that when intraperitoneally administrated to mice, LMTM might not have direct interaction with

tau as shown in vitro test (Fig. 3a, b). The anti-tau activity of LMTM shown in the brain of Tau ${ }^{\text {P301L-BiFC }}$ mice could be the result of indirect regulation of tau pathway, such as decreasing tau phosphorylation or increasing autophagic clearance of aggregated proteins ${ }^{7,46}$. Collectively, our results indicated that levosimendan is a new drug candidate targeting tau oligomerization, suppressing tau phosphorylation and aggregation in Tau ${ }^{\mathrm{P} 301 \mathrm{~L}-\mathrm{BiFC}}$ mice.

\section{Discussion}

Levosimendan was identified 20 year ago and has been treated to patients with acute heart failure ${ }^{47}$. As an inodilator, levosimendan possesses both positive inotropic and vasodilator actions; (i) as a calcium sensitizer, it enhances the sensitivity of contractile proteins to calcium through covalent binding to troponin $\mathrm{C}^{48}$. (ii) as a vasodilator, levosimendan inhibits phosphodiesterase III and opens ATP-dependent $\mathrm{K}+$ channels in smooth muscle cells, leading to arteriolar and venous dilation ${ }^{49}$. In addition to heart, ATPdependent $\mathrm{K}+$ channels present in a number of tissues including brain ${ }^{50}$. In 2010, Roehl et al reported neuroprotective effect of levosimendan in vitro model of traumatic brain injury, which might be associated with the activation of neuronal ATP-dependent $\mathrm{K}+$ channels $^{51,52}$. There are also evidences indicating that the neurorotective effect of levosimendan is associated with its action as a vasodilator. In 2015 , Levijoki et al reported that orally administered levosimendan increased blood volume of the cerebral vessels, reducing mortality and morbidity in rat models of primary and secondary stroke $\mathrm{e}^{53}$. In 2015, Matti Kivikko et al reported a pilot study showing that low dose oral administration of levosimendan increased cerebral blood flow velocities in patients with an earlier ischemic cerebrovascular event, supporting the vasodilatory effect in the brain circulation ${ }^{54}$.

Here our study shows a new molecular mechanism of levosimendan as an anti-tau agent. By modifying tau cysteine residues, levosimendan inhibits disulfide-linked tau oligomerization. Different from LMTM, 
which showed conflicting effects on tau, levosimendan exhibited consistent, inhibitory effect on tau oligomerization in vitro, in cells, and in the brain of mice, suppressing tau aggregation. In tau-BiFC mice, 4 month-administration of levosimendan not only suppressed tau pathology, but also prevented memory

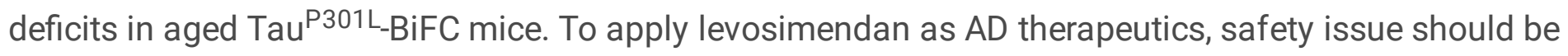
clarified, due to its action as an inodilator. There is strong association between dementia and cardiovascular disease. Cardiovascular insufficiency impairs the function of diverse organs, including the brain, which can worsen a pathology related to dementia ${ }^{55,56}$. For an example, phosphodiesterase III is known to be upregulated in cerebral blood vessels of $A D$ patients due to vascular amyloid burden, and phosphodiesterase III inhibitors have shown protective effects in AD model ${ }^{57,58}$. In that case, the vasodilator-action of levosimendan would be beneficial in improving AD brain function.

Drug repositioning is an attractive drug discovery strategy, which generates new therapeutic value from existing drugs ${ }^{59}$. MB is one of the representative cases of drug repositioning. In 1891, MB was firstly approved to treat malaria with its activity as a chloroquine sensitizer ${ }^{60}$. Then, MB had been approved to treat clinical pain syndromes, psychotic disorders, cyanide poisoning and urinary tract infections ${ }^{61}$. In 2016, MB was approved to treat Methemoglobinemia ${ }^{62}$. In addition to the known medical actions of MB, many studies have compiled wide variety of biological activities of $M B$, which i) inhibits the activity of monoamine oxidase $A^{63}$, nitric oxide synthase ${ }^{64}$, and guanylate cyclase ${ }^{65}$, ii) increases the release of neurotransmitters, such as serotonin and norepinephrine ${ }^{66,67}$, iii) increases cholinergic transmission ${ }^{68}$, iv) inhibits GSK3b and microtubule-affinity regulating kinases ${ }^{69,70}$, and v) promotes autophagic clearance of b-amyloid ${ }^{71}$ and tau ${ }^{72}$. The action of methylene blue on multiple targets in the brain justifies its

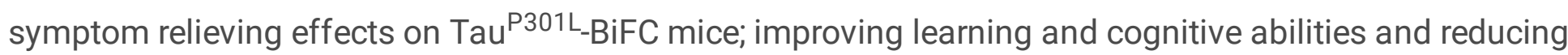
tau phosphorylation and aggregates. However, our in vitro and cell-based data clearly demonstrated that tau oligomer is not the direct therapeutic target of MB or LMTM.

In AD patients, tau oligomers are detected at the early stages of pathogenic cascades ${ }^{14,15,73}$. Therefore, preventing tau oligomerization is an important therapeutic strategy to prevent neuronal loss and memory deficits in AD patients. However, it has been difficult to establish therapeutic strategy preventing tau oligomerization, since tau pathology is linked with a number of cellular processes, which are closely linked to each other. For example, if GSK3b a major tau kinase, is inhibited, other tau kinases activate tau pathology $y^{5,74}$. Therefore, direct modification of tau would be more effective a strategy to prevent tau oligomerization without altering other cellular processes. Our results showed that levosimendan covalently binds to tau cysteine residues and inhibits tau oligomerization preventing tau pathology. Moreover, levosimendan could disassemble tau-tau interactions regardless of its aggregation states. The binding mode between levosimendan and tau should be clarified in future study; how levosimendan dissociates disulfide-linked tau oligomers into monomers; whether levosimendan could distinguish cytosolic tau from microtubule-bound tau. Once levosimendan disassembles tau oligomers into monomers, the monomeric tau would be degraded by proteasome complexes, reducing soluble tau burden ${ }^{75}$. Our results supported this by showing the decreased level of tau in levosimendan-treated tau- 
BiFC cells and primary neurons, together with the reduced level of tau oligomers. Levosimendan displayed robust potency against tau oligomerization and rescued tauopathy-induced cognitive declines

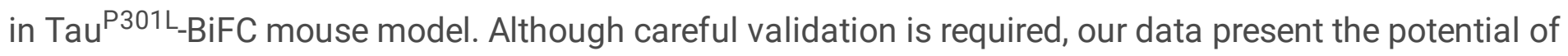
levosimendan as a disease modifying drug for AD.

\section{Materials And Methods}

\section{Source of chemicals}

FDA-approved \& Passed Phase I Drug Library was purchased from Selleckchem. Forskolin and MB were purchased from Sigma, and leucomethylene blue mesylate (LMTM) was purchased from MedChemExpress. Levosimendan and its metabolites, OR-1855 and OR-1896 were purchased from Toronto Research Chemicals Inc. Synthesis and characterization of ${ }^{14} \mathrm{C}$-levosimendan were conducted by Curachem, Inc. according to previously described protocol ${ }^{76}$. The characterization of ${ }^{14} \mathrm{C}$-levosimendan is provided in in the Supplementary Materials.

\section{Preparation of tauK18 fragments}

TauK18 and tauK $18^{\mathrm{P} 301 \mathrm{~L}}$ fragments were expressed and purified according to previously described protocols ${ }^{19,77}$. 6xHis-tagged tauK18 and tauK18 ${ }^{\mathrm{P} 301 \mathrm{~L}}$ were expressed in E.coli BL21 (DE3) and purified by using Ni-NTA (QIAGEN) column chromatography. The purified protein was dissolved in PBS (pH 7.4). Peptide-synthesis and characterization of each repeat domain (R1-R4) were conducted by GL Biochem. ESI-MS characterization of the peptides is provided in the Supplementary materials.

\section{Tau-BiFC cell culture and the treatment of FK or tauK18 $8^{\mathrm{P} 301 \mathrm{~L}}$}

HEK293 Tau-BiFC cells were maintained in Dulbecco's modified eagle medium (DMEM) supplemented with $10 \%$ fetal bovine serum (FBS), 100 units/mL penicillin, $100 \mu \mathrm{g} / \mathrm{mL}$ streptomycin and $100 \mu \mathrm{g} / \mathrm{mL}$ Geneticin (G418) in humidified atmosphere containing $5 \% \mathrm{CO} 2$ at $37^{\circ} \mathrm{C}$. Tau-BiFC cells were activated by the treatment of FK $(30 \mathrm{mM})$ or tauK18 ${ }^{\mathrm{P} 301 \mathrm{~L}}(5 \mathrm{mg} / \mathrm{mL})$.

\section{FDA-approved \& Passed Phase I Drug Library screening and high-contents image analysis}

For screening of FDA-approved \& Passed Phase I Drug Library, tau-BiFC cells were plated on $\mu$-clear 384well plates. The next day, the cells were co-treated with forskolin and 1,018 library compounds at $10 \mu \mathrm{M}$ concentration. MB and LMTM $(3 \mu \mathrm{M})$ were used as a positive control. After $46 \mathrm{hrs}$, nuclei were counterstained with Hoechst (Invitrogen). BiFC ( $\lambda_{\mathrm{ex}}=460-490 \mathrm{~nm}$ and $\left.\lambda_{\mathrm{em}}=500-550 \mathrm{~nm}\right)$ and Hoechst $\left(\lambda_{\mathrm{ex}}=355-385 \mathrm{~nm}\right.$ and $\lambda_{\mathrm{em}}=430-500 \mathrm{~nm}$ ) fluorescence images were acquired automatically by using Operetta ${ }^{\circledR}$ (PerkinElmer). BiFC-fluorescence intensity and the number of nuclei were quantified using the Harmony 3.1 software (PerkinElmer). Integrated tau-BiFC intensity represents Area of selected cell X Mean fluorescence intensity. The means and standard deviations (S.D.) of BiFC fluorescence intensities and the number of nuclei were plotted using GraphPad Prism. 


\section{Flow cytometry analysis}

Tau-BiFC cells, grown in 6-well plates, were treated with MB, LMTM $(5 \mu \mathrm{M})$, or levosimendan $(15 \mu \mathrm{M})$, followed by the treatment of tauK18 $8^{\mathrm{P} 301 \mathrm{~L}}(5 \mu \mathrm{g} / \mathrm{mL})$. After $24 \mathrm{hrs}$, BiFC cells were collected and subjected to flow cytometry analysis, using BD FACSLyric ${ }^{\text {TM }}$ cytometer (BD Biosciences). A total of 50,000 events were acquired per sample. All samples were gated using the same gating tree and gate positions; side scatter area (SSC-A) vs. forward scatter area (FSC-A) for viable and singlet cell populations. BiFC fluorescence was excited by $488 \mathrm{~nm}$ wave laser and collected through a 527/32 band-pass filter.

\section{Preparation of Tau-BiFC cell lysates}

Tau-BiFC cells, grown in 6-well plates, were treated with $\operatorname{MB}, \operatorname{LMTM}(0.5,1.5,5 \mu \mathrm{M})$, or levosimendan $(5$, $15,45 \mu \mathrm{M})$ followed by tauK $18^{\mathrm{P} 301 \mathrm{~L}}(5 \mu \mathrm{g} / \mathrm{mL})$ activation. After $36 \mathrm{hrs}$, tau-BiFC cells were washed with PBS and lysed in CelLytic M lysis reagent (Sigma) containing protease/phosphatase inhibitor cocktail (Sigma).

\section{Tau-immunoblot analysis of reducing and non-reducing SDS-PAGE gels}

For reducing SDS-PAGE analysis, cell or brain lysates were mixed with 4x Laemmli buffer containing $10 \%$ $\beta$-mercaptoethanol (BME) and boiled at $97^{\circ} \mathrm{C}$ for $5 \mathrm{~min}$. For non-reducing SDS-PAGE analysis, cell or brain lysates were mixed with $4 \mathrm{x}$ Laemmli buffer without BME. For immunoblot analysis, $10 \mu \mathrm{g}$ of each lysate was separated into 10\% SDS-PAGE gel and transferred to PVDF membrane. The levels of total tau and phosphorylated tau were detected by anti-tau antibody; 2B11 (IBL), Tau5 (Abcam), pSer199 (Abcam), pSer396 (Abcam), and pThr205 (Abcam). Band intensity was quantified using Image J software $(\mathrm{NIH})$.

\section{Primary neuron culture and preparation of the lysates}

Primary hippocampal neurons were isolated from day 18 embryonic Sprague-Dawley rat brains as described previously ${ }^{78}$. The neurons were seeded at a density of $3.5 \times 10^{5}$ cells/well on a poly-D-lysinecoated 6-well plate and maintained in the neurobasal medium at $37^{\circ} \mathrm{C}$ in a humidified atmosphere of $5 \%$ $\mathrm{CO}_{2}$. The neurobasal medium contains $2 \% \mathrm{~B} 27$ supplement, $0.5 \mathrm{mM}$ glutamax, 100 units $/ \mathrm{mL}$ penicillin, and $100 \mu \mathrm{g} / \mathrm{mL}$ streptomycin. Every 3 days, $50 \%$ of the medium was replaced with the fresh neurobasal medium. At DIV10, neurons were incubated with MB, LMTM $(3 \mu \mathrm{M})$, or levosimendan $(10 \mu \mathrm{M})$ in the presence of tauK18 ${ }^{\text {P301L }}(10 \mu \mathrm{g} / \mathrm{mL})$ for $48 \mathrm{hrs}$. Then, the neurons were washed with PBS and lysed in CelLytic M lysis reagent (Sigma) containing protease/phosphatase inhibitor cocktail (Sigma).

\section{NeuO stain and analysis of neurite lengths}

At DIV10, neurons grown on a 96-well plate were treated with MB, LMTM $(3 \mu \mathrm{M})$, or levosimendan (10 $\mu \mathrm{M})$ followed by tauK $18^{\mathrm{P} 301 \mathrm{~L}}$ activation. After $48 \mathrm{hrs}$, the neurons were stained with NueO $(50 \mathrm{nM})$, a neuron selective probe ${ }^{41}$. NeuO-fluorescence $\left(\lambda_{\mathrm{ex}}=460-490 \mathrm{~nm}\right.$ and $\left.\lambda_{\mathrm{em}}=518-580 \mathrm{~nm}\right)$ images were 
acquired by using Operetta ${ }^{\circledR}$ (PerkinElmer). The maximal length of neurites was quantified by Harmony 3.1 software (PerkinElmer).

\section{TauK18 oligomerization assay in vitro}

To induce tau oligomerization, tauK18 $(0.5 \mathrm{mg} / \mathrm{mL}$ dissolved in PBS, pH7.4) was incubated with each drug in the presence of DTT $(100 \mu \mathrm{M})$ and heparin $(0.1 \mathrm{mg} / \mathrm{mL})$ at RT for $5 \mathrm{hrs}$ with vigorous shaking. Then, tau oligomers were separated on 4-20\% SDS-PAGE gels under reducing and non-reducing condition and visualized by Coomassie blue stain.

\section{In vitro tau aggregation and disaggregation assays.}

To evaluate the inhibitory effect of drugs on tau aggregation, tauK18 protein $(0.5 \mathrm{mg} / \mathrm{mL})$ was incubated with each drug at various concentration in the presence of DTT $(100 \mu \mathrm{M})$ and heparin $(0.1 \mathrm{mg} / \mathrm{mL})$ at $37^{\circ} \mathrm{C}$ for 5 days, with vigorous shaking $(220 \mathrm{rpm})$. For tau disaggregation assay, the preformed aggregation-mixture of tauK 18 was incubated with each drug at $37^{\circ} \mathrm{C}$ for 4 days, with vigorous shaking (220 rpm). At the final day of the incubation, the level of b-sheet aggregates was evaluated by thioflavin $S$ (ThS) assay. For ThS assay, $5 \mu \mathrm{L}$ of each mixture was transferred to a black 384-well plate with $45 \mu \mathrm{L}$ of PBS containing $10 \mu \mathrm{M}$ ThS. ThS fluorescence $\left(\lambda_{\mathrm{ex}}=430 \mathrm{~nm}\right.$, and $\left.\lambda_{\mathrm{em}}=500 \mathrm{~nm}\right)$ was measured by using a Flexstation2 spectrophotometer (Molecular Devices).

\section{Transmission electron microscopy (TEM)}

Samples were placed onto carbon-coated copper electron microscopy grids, and then negatively stained with $2 \%(\mathrm{w} / \mathrm{v})$ aqueous uranyl acetate for $1 \mathrm{~min}$. For imaging of tau filaments (Fig. 3D), the grids were observed using a JEM-1011 transmission electron microscope (JEOL) at the acceleration voltage of 80 kV. For imaging of tau filaments and oligomers (Fig. 3F and Fig. 4E), the grids were observed using Tecni G2 F20 transmission electron microscope (FEl) at the acceleration voltage of $120 \mathrm{kV}$. Eight to twelve random images from each experimental condition were captured by the operator as a blind observer.

\section{Autoradiography of ${ }^{14} \mathrm{C}$-levosimendan}

TauK18 $(1 \mathrm{mg} / \mathrm{mL}, 72 \mu \mathrm{M})$ was incubated with ${ }^{14} \mathrm{C}$-levosimendan $(720 \mu \mathrm{M})$ in PBS containing $5 \%$ of DMSO at RT for $2 \mathrm{hrs}$. Then, the mixture was separated on 15\% SDS-PAGE gel under non-reducing condition and stained with Coomassie blue. For autoradiography, the SDS-PAGE gel was transferred to PVDF membrane and scanned by Typhoon FLA 7000 IP (GE Healthcare).

\section{MALDI-TOF analysis of R1-R4 peptides}

Each repeat domain (R1- R4, $150 \mathrm{mM}$ ) was incubated with levosimendan (molar ratio 1:10 of a tau repeat domain: levosimendan) at RT for $37 \mathrm{hrs}$. The samples were mixed with matrix solution $(10 \mathrm{mg} / \mathrm{mL}$ of Sinapinic acid in $0.1 \%(\mathrm{v} / \mathrm{v})$ trifluoroacetic acid/CAN) at a ratio $1: 1$. The mixtures were directly spotted onto the MALDI target and dried. Mass spectra were acquired in reflection/linear positive ion mode in the 
$\mathrm{m} / \mathrm{z}$ range of 2,000-100,000 using an Ultraflex III TOF/TOF mass spectrometer controlled by Flex Control 3.0 (Bruker Daltonics). Default operating conditions are as follows: ion source 1, $25.0 \mathrm{kV}$; ion source 2, $23.0 \mathrm{kV}$; lens voltage, $6.0 \mathrm{kV}$; laser repetition rate $=100 \mathrm{~Hz}$. All spectra were generated automatically in the instrument software and based on averaging 1000 shots from 10 non-overlapping positions (100 shots/position).

\section{Animal studies}

\section{Tau ${ }^{\text {P301L-BiFC mice }}$}

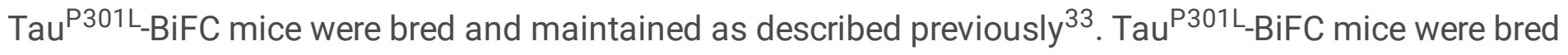
with C57BL/6N mice and maintained in pathogen-free facilities. Their heterogeneous offspring and wildtype littermates were used in this study. All mice were allocated randomly for experiments, but groups were counterbalanced for animal sex and group average body weight. Animal protocols followed the principles and practices outlined in the approved guidelines by the Institutional Animal Care and Use Committee of the Korea Institute of Science and Technology. All animal experiments were approved by the Korea Institute of Science and Technology.

\section{Drug administration to Tau ${ }^{\mathrm{P} 301 \mathrm{~L}-\mathrm{BiFC} \text { mice }}$}

For drug administration, LMTM and levosimendan were dissolved in PBS containing $40 \%$ polyethylene

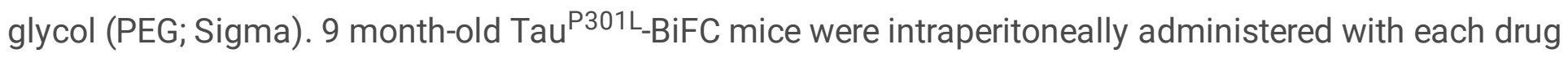
for 4 months, three times a week, with $5 \mathrm{mg} / \mathrm{kg}$ dosage ( $\mathrm{n}=11$ per group). Vehicle-treated Tau ${ }^{\text {P301L }}$-BiFC mice and age-matched wild type mice were used as control groups. Behavioral assessments were conducted as described below for one month from the end of drug administration. All mice were sacrificed at the age of 14 months for pathological analysis.

\section{Behavioral tests}

Novel object recognition. Novel object recognition test was performed as described previously ${ }^{79}$. Briefly, for the habituation, mice ( $\mathrm{n}=9$ per group) were individually placed in the center of an open field arena (40 $\times 40 \mathrm{~cm}$ ) and allowed to freely explore for $15 \mathrm{~min}$. Next day, in the training trial (familiarization phase), mice were allowed to explore two identical objects in the open field for $10 \mathrm{~min}$. In the testing trial (recognition phase) performed 24 hours later, one familiar object was changed for a novel object which was different in color and shape. Mice were allowed to explore the objects for $10 \mathrm{~min}$. The exploration time for the familiar (old) or the new (novel) object during the recognition phase was recorded using Noldus EthoVision XT video tracking system. Memory was operationally defined by the recognition index calculated by dividing the time an animal spent exploring the novel object or old object by the total time spent exploring objects in the testing period. The exploration time was measured when the mouse was pointing towards the object in the vicinity of the object. 
Y-maze. Mice ( $\mathrm{n}=11$ per group) were tested for spontaneous alternation behavior in a Y-shaped maze (60 $\mathrm{cm}$ in length of each arm, $20 \mathrm{~cm}$ in depth) using standard protocol ${ }^{80}$. Spontaneous alternation for 8 mins was calculated as the proportion of alternations. Alteration (\%) was defined as consecutive entries in three different arms (ABC), divided by the number of possible alterations (total arm entries minus 2$)^{81}$.

Passive avoidance. Passive avoidance test was performed as described previously ${ }^{82}$ with the following modifications. Mice ( $n=8$ per group) were adapted in the passive avoidance chamber (Gemini) for 10 min, and then were returned to their home cages. The chamber is composed of a light compartment and a dark compartment separated by a connecting gate. The following day, mice were placed in the light compartment, and the gate was opened after 30 seconds. When mice entered the dark compartment, the gate was closed and an electrical foot shock ( $3 \mathrm{~mA}$ ) was delivered for $2 \mathrm{sec}$. Mice were left in the dark compartment for $30 \mathrm{sec}$ after the foot shock to enable association of the environment with the aversive stimulus. Mice were then returned to their home cages. The following day, mice were placed in the light compartment again, and the gate was opened after 30 seconds. The step-through latency, the time required for mice to enter the dark compartment, was measured up to $540 \mathrm{sec}$.

\section{Preparation of brain tissue slices}

Mice were perfused with $0.9 \%$ saline and fixed with PBS (pH 7.4) containing $4 \%$ paraformaldehyde (Sigma). Brains were extracted and fixed in PBS containing $4 \%$ paraformaldehyde at $4^{\circ} \mathrm{C}$ for $48 \sim 72 \mathrm{hrs}$. For cryoprotection, the brains were transferred to PBS containing $30 \%$ sucrose solution and incubated at $4^{\circ} \mathrm{C}$ until they sunk. For cryosectioning, the brains were embedded with O.C.T (Tissue-TEK), and cut serially using a cryostat (CM1860UV, Leica). 30- $\mu \mathrm{m}$ thick tissue slices were maintained in PBS containing $0.05 \%$ sodium azide at $4^{\circ} \mathrm{C}$.

\section{Sudan Black B stain and BiFC fluorescence imaging}

Brain tissue section ( $\mathrm{n}=8$ per group) were mounted onto slides. To reduce autofluorescence, brain tissue sections were stained with Sudan Black B solution ( $70 \%$ ethanol containing $0.05 \%$ Sudan Black B) for 10 min, washed three times with PBS containing $0.1 \%$ Triton X-100 (Sigma), and then washed with distilled water. For counter-staining of nuclei, brain tissues were stained with Hoechst $(0.5 \mu \mathrm{g} / \mathrm{mL})$ for $30 \mathrm{~min}$. BiFC fluorescence $\left(\lambda_{\mathrm{ex}}=460-490 \mathrm{~nm}\right.$ and $\left.\lambda_{\mathrm{em}}=500-550 \mathrm{~nm}\right)$ images were acquired using Axio Scan. Z1 (ZEISS). For quantitative analysis, Mean BiFC-fluorescence intensity was measured in somatosensory cortex (layer $\mathrm{V}$ ) and hippocampal CA1 from AP;-1.82. To normalize the background fluorescence between the brain samples, habenular region was used as an internal control. Using image $J$ software $(\mathrm{NIH})$, the region of interest was masked, and the fluorescence intensity value was calculated. Data were shown as the average and standard deviation of Mean fluorescence intensities of brain images from six to eight animals.

\section{Immunofluorescence analysis of brain tissue slices}


For immunofluorescence image analysis, brain tissue slices ( $n=6 \sim 8$ per group) were stained with AT8 (pS202/T205) antibody (1:200, Invitrogen). The primary antibody was detected using Alexa Flour 633conjugated anti-mouse antibody (1:500, abcam). Fluorescence images $\left(\lambda_{\mathrm{ex}}=620-640 \mathrm{~nm}\right.$ and $\lambda_{\mathrm{em}}=650$ $700 \mathrm{~nm}$ ) were acquired using Axio Scan. Z1 (ZEISS).

\section{Preparation of RIPA-soluble and insoluble brain lysates}

Brains were weighed and suspended in RIPA lysis buffer (Sigma) containing protease and phosphatase inhibitor cocktails. Then, tissues were mechanically disrupted using a cordless mortar and pestle (Sigma), and incubated at $4^{\circ} \mathrm{C}$ for $2 \mathrm{hrs}$, with shaking. The homogenates were centrifuged at $20,000 \mathrm{~g}$ at $4^{\circ} \mathrm{C}$ for 20 min. The supernatants were collected as RIPA-soluble fractions and stored at $-80^{\circ} \mathrm{C}$. To prepare RIPAinsoluble fractions, the remaining pellets were washed once with RIPA lysis buffer containing $1 \mathrm{M}$ sucrose, and then resuspended in $2 \%$ SDS solution ( $1 \mathrm{~mL}$ per gram of tissue) and incubated at RT for 1 $\mathrm{hr}$. The mixtures were centrifuged at $20,000 \mathrm{~g}$ for $1 \mathrm{~min}$ at RT. The supernatant was collected as RIPAinsoluble fractions and preserved at $-80^{\circ} \mathrm{C}$.

\section{Statistics}

All data in quantitative analysis were presented as mean \pm S.D. or S.E.M.. Unpaired $t$ test was performed when two groups were compared. One-way ANOVA or two-way ANOVA was performed when multiple groups were compared, depending on the number of independent variables. Statistical analysis was performed using GraphPad Prism 6 (GraphPad Software).

\section{Declarations}

\section{Author contributions}

Y.K.K. designed the study and provided theoretical guidance; S.L., S.S., H.E.L., J.Y.S., Y.S., G.H.L., H.A., N.L., K.H.K., and H.J., performed the experiments; S.L., S.S., and Y.K.K. interpreted the data and wrote the manuscript; and S.L., S.S., B.Y.Y., S.M.L., J.S.L., A.N.P., and Y.K.K. analyzed the data and revised the manuscript. All authors reviewed the final version of the manuscript.

\section{Competing interests}

The authors declare that they have no competing interests.

\section{Data and materials availability}

All data associated with this study are present in the paper or the Supplementary Materials.

\section{References}


1 Kolarova, M., García-Sierra, F., Bartos, A., Ricny, J. \& Ripova, D. Structure and pathology of tau protein in Alzheimer disease. International journal of Alzheimer's disease 2012 (2012).

2 Kontaxi, C., Piccardo, P. \& Gill, A. C. Lysine-directed post-translational modifications of tau protein in Alzheimer's disease and related Tauopathies. Frontiers in molecular biosciences 4, 56 (2017).

3 Kadavath, H. et al. Tau stabilizes microtubules by binding at the interface between tubulin heterodimers. Proceedings of the National Academy of Sciences 112, 7501-7506 (2015).

4 Paglini, G., Peris, L., Mascotti, F., Quiroga, S. \& Caceres, A. Tau protein function in axonal formation. Neurochemical research 25, 37-42 (2000).

5 Stoothoff, W. H. \& Johnson, G. V. Tau phosphorylation: physiological and pathological consequences. Biochimica et Biophysica Acta (BBA)-Molecular Basis of Disease 1739, 280-297 (2005).

6 Martin, L., Latypova, X. \& Terro, F. Post-translational modifications of tau protein: implications for Alzheimer's disease. Neurochemistry international 58, 458-471 (2011).

7 Chesser, A., Pritchard, S. \& Johnson, G. V. Tau clearance mechanisms and their possible role in the pathogenesis of Alzheimer disease. Frontiers in neurology 4, 122 (2013).

8 Serrano-Pozo, A., Frosch, M. P., Masliah, E. \& Hyman, B. T. Neuropathological alterations in Alzheimer disease. Cold Spring Harbor perspectives in medicine 1, a006189 (2011).

9 Lee, V. M., Goedert, M. \& Trojanowski, J. Q. Neurodegenerative tauopathies. Annual review of neuroscience 24, 1121-1159 (2001).

10 Himmelstein, D. S., Ward, S. M., Lancia, J. K., Patterson, K. R. \& Binder, L. I. Tau as a therapeutic target in neurodegenerative disease. Pharmacology \& therapeutics 136, 8-22 (2012).

11 Shafiei, S. S., Guerrero-Muñoz, M. J. \& Castillo-Carranza, D. L. Tau oligomers: cytotoxicity, propagation, and mitochondrial damage. Frontiers in aging neuroscience 9, 83 (2017).

12 Lasagna-Reeves, C. A. et al. Tau oligomers impair memory and induce synaptic and mitochondrial dysfunction in wild-type mice. Molecular neurodegeneration 6, 39 (2011).

13 Kaniyappan, S., Chandupatla, R. R., Mandelkow, E.-M. \& Mandelkow, E. Extracellular low-n oligomers of tau cause selective synaptotoxicity without affecting cell viability. Alzheimer's \& Dementia 13, 1270-1291 (2017).

14 Maeda, S. et al. Granular tau oligomers as intermediates of tau filaments. Biochemistry 46, 38563861 (2007).

15 Maeda, S. et al. Increased levels of granular tau oligomers: an early sign of brain aging and Alzheimer's disease. Neurosci Res 54, 197-201, doi:10.1016/j.neures.2005.11.009 (2006). 
16 Berger, Z. et al. Accumulation of pathological tau species and memory loss in a conditional model of tauopathy. The Journal of neuroscience : the official journal of the Society for Neuroscience 27, 3650-3662, doi:10.1523/JNEUROSCI.0587-07.2007 (2007).

17 Clavaguera, F. et al. Transmission and spreading of tauopathy in transgenic mouse brain. Nature cell biology 11, 909-913, doi:10.1038/ncb1901 (2009).

18 Frost, B., Jacks, R. L. \& Diamond, M. I. Propagation of tau misfolding from the outside to the inside of a cell. The Journal of biological chemistry 284, 12845-12852, doi:10.1074/jbc.M808759200 (2009).

19 Kim, D. et al. Identification of disulfide cross-linked tau dimer responsible for tau propagation. Scientific reports 5, 15231 (2015).

20 Haque, M., Lim, S., Kim, D., Kim, D. J. \& Kim, Y. K. Intracellular tau modifications and cell-based sensors for monitoring tau aggregation.

21 Wischik, C. M., Bentham, P., Wischik, D. J. \& Seng, K. M. 03-04-07: Tau aggregation inhibitor (TAI) therapy with rember ${ }^{\mathrm{T}}$ arrests disease progression in mild and moderate Alzheimer's disease over 50 weeks. Alzheimer's \& Dementia 4, T167-T167 (2008).

22 Akoury, E. et al. Mechanistic basis of phenothiazine-driven inhibition of Tau aggregation. Angewandte Chemie International Edition 52, 3511-3515 (2013).

23 Crowe, A. et al. Aminothienopyridazines and methylene blue affect Tau fibrillization via cysteine oxidation. Journal of Biological Chemistry 288, 11024-11037 (2013).

24 Walker, S., Ullman, O. \& Stultz, C. M. Using intramolecular disulfide bonds in tau protein to deduce structural features of aggregation-resistant conformations. Journal of Biological Chemistry 287, 9591 9600 (2012).

25 Soeda, Y. et al. Methylene blue inhibits formation of tau fibrils but not of granular tau oligomers: A plausible key to understanding failure of a clinical trial for Alzheimer's disease. Journal of Alzheimer's Disease 68, 1677-1686 (2019).

26 Wilcock, G. K. et al. Potential of low dose leuco-methylthioninium bis (hydromethanesulphonate) (LMTM) monotherapy for treatment of mild Alzheimer's disease: Cohort analysis as modified primary outcome in a phase III clinical trial. Journal of Alzheimer's Disease 61, 435-457 (2018).

27 Gauthier, S. et al. Efficacy and safety of tau-aggregation inhibitor therapy in patients with mild or moderate Alzheimer's disease: a randomised, controlled, double-blind, parallel-arm, phase 3 trial. The Lancet 388, 2873-2884 (2016). 
28 Biernat, J., Gustke, N., Drewes, G. \& Mandelkow, E. Phosphorylation of Ser262 strongly reduces binding of tau to microtubules: distinction between PHF-like immunoreactivity and microtubule binding. Neuron 11, 153-163 (1993).

29 Lindwall, G. \& Cole, R. D. Phosphorylation affects the ability of tau protein to promote microtubule assembly. Journal of Biological Chemistry 259, 5301-5305 (1984).

30 Min, S.-W. et al. Acetylation of tau inhibits its degradation and contributes to tauopathy. Neuron 67, 953-966 (2010).

31 Reynolds, M. R., Berry, R. W. \& Binder, L. I. Site-specific nitration and oxidative dityrosine bridging of the $\tau$ protein by peroxynitrite: implications for Alzheimer's disease. Biochemistry 44, 1690-1700 (2005).

32 Tak, H. et al. Bimolecular fluorescence complementation; lighting-up tau-tau interaction in living cells. PloS one 8, e81682, doi:10.1371/journal.pone.0081682 (2013).

33 Shin, S. et al. Visualization of soluble tau oligomers in TauP301L-BiFC transgenic mice demonstrates the progression of tauopathy. Progress in Neurobiology, 101782 (2020).

34 Jeong, H. et al. Pan-HDAC Inhibitors Promote Tau Aggregation by Increasing the Level of Acetylated Tau. International journal of molecular sciences 20, 4283 (2019).

35 Lim, S. et al. Monitoring of intracellular tau aggregation regulated by OGA/OGT inhibitors. International journal of molecular sciences 16, 20212-20224 (2015).

36 Choi, W. H. et al. Open-gate mutants of the mammalian proteasome show enhanced ubiquitinconjugate degradation. Nature communications 7, 1-12 (2016).

37 Wang, H.-H. et al. Forskolin induces hyperphosphorylation of tau accompanied by cell cycle reactivation in primary hippocampal neurons. Molecular Neurobiology 55, 696-706 (2018).

38 Yan, K., Gao, L. N., Cui, Y. L., Zhang, Y. \& Zhou, X. The cyclic AMP signaling pathway: Exploring targets for successful drug discovery. Molecular medicine reports 13, 3715-3723 (2016).

39 Yamada, K. et al. Analysis of in vivo turnover of tau in a mouse model of tauopathy. Molecular neurodegeneration 10, 1-9 (2015).

40 Sahara, N. et al. Assembly of two distinct dimers and higher-order oligomers from full-length tau. European Journal of Neuroscience 25, 3020-3029 (2007).

$41 \mathrm{Er}, \mathrm{J}$. C. et al. NeuO: a fluorescent chemical probe for live neuron labeling. Angewandte Chemie International Edition 54, 2442-2446 (2015).

42 Taniguchi, S. et al. Inhibition of heparin-induced tau filament formation by phenothiazines, polyphenols, and porphyrins. Journal of Biological Chemistry 280, 7614-7623 (2005). 
43 Abskharon, R. et al. Crystal structure of a conformational antibody that binds tau oligomers and inhibits pathological seeding by extracts from donors with Alzheimer's disease. Journal of Biological Chemistry, jbc. RA120. 013638 (2020).

44 Antila, S. et al. Pharmacokinetics of levosimendan and its active metabolite OR-1896 in rapid and slow acetylators. European journal of pharmaceutical sciences 23, 213-222 (2004).

45 Takahashi, R., Talukder, M. H. \& Endoh, M. Effects of OR-1896, an active metabolite of levosimendan, on contractile force and aequorin light transients in intact rabbit ventricular myocardium. Journal of cardiovascular pharmacology 36, 118-125 (2000).

46 Dolan, P. J. \& Johnson, G. V. The role of tau kinases in Alzheimer's disease. Current opinion in drug discovery \& development 13, 595 (2010).

47 Haikala, H. et al. Cardiac troponin $\mathrm{C}$ as a target protein for a novel calcium sensitizing drug, levosimendan. Journal of molecular and cellular cardiology 27, 1859-1866 (1995).

48 Pollesello, P. et al. Binding of a new Ca2+ sensitizer, levosimendan, to recombinant human cardiac troponin C. A molecular modelling, fluorescence probe, and proton nuclear magnetic resonance study. Journal of Biological Chemistry 269, 28584-28590 (1994).

49 Yildiz, O. Vasodilating mechanisms of levosimendan: involvement of K+ channels. Journal of pharmacological sciences 104, 1-5 (2007).

50 Miki, T. et al. ATP-sensitive $\mathrm{K}+$ channels in the hypothalamus are essential for the maintenance of glucose homeostasis. Nature neuroscience 4, 507-512 (2001).

51 Roehl, A. B. et al. Neuroprotective properties of levosimendan in an in vitro model of traumatic brain injury. BMC neurology 10, 97 (2010).

52 Roehl, A. B. et al. The effects of levosimendan on brain metabolism during initial recovery from global transient ischaemia/hypoxia. BMC neurology 12, 1-10 (2012).

53 Levijoki, J. et al. Levosimendan alone and in combination with valsartan prevents stroke in Dahl salt-sensitive rats. European Journal of Pharmacology 750, 132-140 (2015).

54 Kivikko, M. et al. Oral levosimendan increases cerebral blood flow velocities in patients with a history of stroke or transient ischemic attack: a pilot safety study. Current Therapeutic Research 77, 46-51 (2015).

55 Justin, B. N., Turek, M. \& Hakim, A. M. Heart disease as a risk factor for dementia. Clinical epidemiology 5, 135 (2013). 
56 de La Torre, J. C. Cardiovascular risk factors promote brain hypoperfusion leading to cognitive decline and dementia. Cardiovascular psychiatry and neurology 2012 (2012).

57 Park, S. H. et al. Protective effect of the phosphodiesterase III inhibitor cilostazol on amyloid $\beta-$ induced cognitive deficits associated with decreased amyloid $\beta$ accumulation. Biochemical and biophysical research communications 408, 602-608 (2011).

58 Maki, T. et al. Phosphodiesterase III inhibitor promotes drainage of cerebrovascular $\beta$-amyloid. Annals of clinical and translational neurology 1, 519-533 (2014).

59 Pushpakom, S. et al. Drug repurposing: progress, challenges and recommendations. Nature reviews Drug discovery 18, 41-58 (2019).

60 Ehrlich, P. \& Guttmann, P. Ueber die wirkung des methylenblau bei malaria. Berliner klinische Wochhenschrift 28, 953-956 (1891).

61 Ginimuge, P. R. \& Jyothi, S. Methylene blue: revisited. Journal of anaesthesiology, clinical pharmacology 26, 517 (2010).

62 Boylston, M. \& Beer, D. Methemoglobinemia: a case study. Critical care nurse 22, 50-55 (2002).

63 Ramsay, R., Dunford, C. \& Gillman, P. Methylene blue and serotonin toxicity: inhibition of monoamine oxidase A (MAO A) confirms a theoretical prediction. British journal of pharmacology 152, 946-951 (2007).

64 Lomniczi, A., Cebral, E., Canteros, G., McCann, S. M. \& Rettori, V. Methylene blue inhibits the increase of inducible nitric oxide synthase activity induced by stress and lipopolysaccharide in the medial basal hypothalamus of rats. Neuroimmunomodulation 8, 122-127 (2000).

65 Farina, J. A., Junior, A. C. C., da Silva, M. F. \& Evora, P. R. B. Guanylate cyclase inhibition by methylene blue as an option in the treatment of vasoplegia after a severe burn. A medical hypothesis. Medical science monitor: international medical journal of experimental and clinical research 18, HY13 (2012).

66 Wegener, G., Volke, V. \& Rosenberg, R. Endogenous nitric oxide decreases hippocampal levels of serotonin and dopamine in vivo. British journal of pharmacology 130, 575-580 (2000).

67 Paya, D., Gray, G. A. \& Stoclet, J.-C. Effects of methylene blue on blood pressure and reactivity to norepinephrine in endotoxemic rats. Journal of cardiovascular pharmacology 21, 926-930 (1993).

68 Oz, M., Lorke, D. E., Hasan, M. \& Petroianu, G. A. Cellular and molecular actions of methylene blue in the nervous system. Medicinal research reviews 31, 93-117 (2011). 

intracerebral hemorrhage in rats through the PI3K/Akt/GSK3 $\beta$ signaling pathway. Journal of cellular physiology 234, 5304-5318 (2019).

70 Sun, W. et al. Attenuation of synaptic toxicity and MARK4/PAR1-mediated Tau phosphorylation by methylene blue for Alzheimer's disease treatment. Scientific reports 6, 34784 (2016).

71 Medina, D. X., Caccamo, A. \& Oddo, S. Methylene blue reduces A $\beta$ levels and rescues early cognitive deficit by increasing proteasome activity. Brain pathology 21, 140-149 (2011).

72 Congdon, E. E. et al. Methylthioninium chloride (methylene blue) induces autophagy and attenuates tauopathy in vitro and in vivo. Autophagy 8, 609-622 (2012).

73 Berger, Z. et al. Accumulation of pathological tau species and memory loss in a conditional model of tauopathy. Journal of Neuroscience 27, 3650-3662 (2007).

74 Engmann, O. \& Giese, K. P. Crosstalk between Cdk5 and GSK3ß: Implications for Alzheimer's disease. Frontiers in molecular neuroscience 2, 2 (2009).

75 Ciechanover, A. \& Kwon, Y. T. Degradation of misfolded proteins in neurodegenerative diseases: therapeutic targets and strategies. Experimental \& molecular medicine 47, e147-e147 (2015).

76 Sandell, E.-P. et al. Pharmacokinetics of levosimendan in healthy volunteers and patients with congestive heart failure. Journal of cardiovascular pharmacology 26, 57-62 (1995).

77 Haque, M. M. et al. Inhibition of tau aggregation by a rosamine derivative that blocks tau intermolecular disulfide cross-linking. Amyloid 21, 185-190 (2014).

$78 \mathrm{Kim}, \mathrm{S}$. et al. Functional interaction of neuronal Cav1. 3 L-type calcium channel with ryanodine receptor type 2 in the rat hippocampus. Journal of Biological Chemistry 282, 32877-32889 (2007).

79 Leger, M. et al. Object recognition test in mice. Nature protocols 8, 2531-2537 (2013).

80 King, D. L. \& Arendash, G. W. Behavioral characterization of the Tg2576 transgenic model of Alzheimer's disease through 19 months. Physiology \& behavior 75, 627-642 (2002).

81 Hsiao, K. K. et al. Age-related CNS disorder and early death in transgenic FVB/N mice overexpressing Alzheimer amyloid precursor proteins. Neuron 15, 1203-1218 (1995).

82 Van der Poel, A. Ethological study of the behaviour of the albino rat in a passive-avoidance test. Acta physiologica et pharmacologica Neerlandica 14, 503 (1967).

\section{Figures}


a

Day 1 (1) Tau-BiFC cell plating on 384 well plates

Day 2 (2) Co-treatment of compound library with Forskolin

(3) Tum-ON Tau-B:FC by Forskolin ( $46 \mathrm{~h}$ )

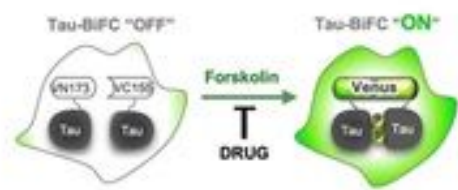

Day 4 (4) Nuclei stain with Hoechst ( $2 \mathrm{~h})$

(5) Image acquisition \& analysis

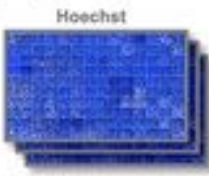

C

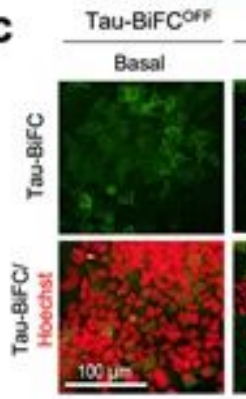

f
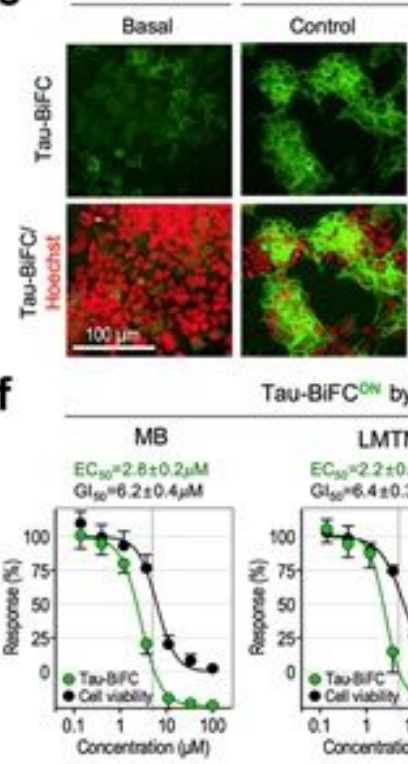

Tau-BiFC ${ }^{\mathrm{ON}}$ by Forskolin

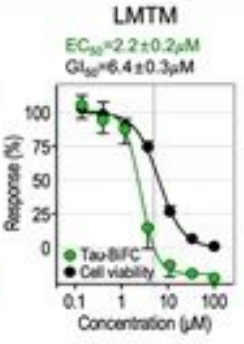

Tau-BiFC ${ }^{\text {os }}$ by Forskolin
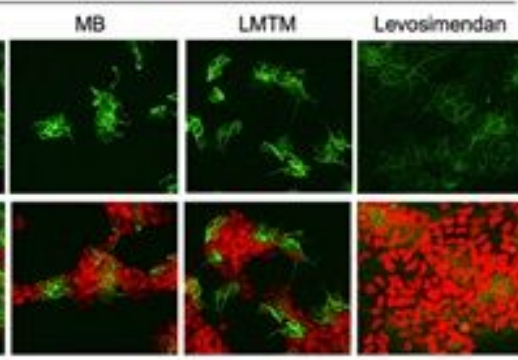

d

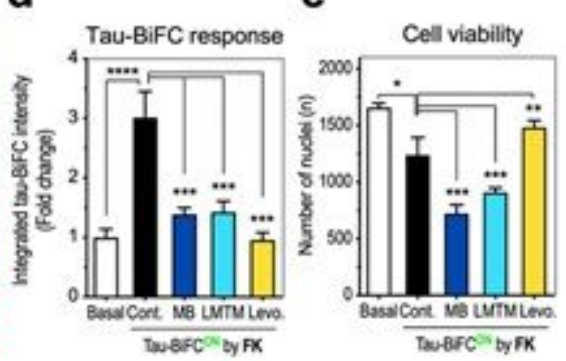

g
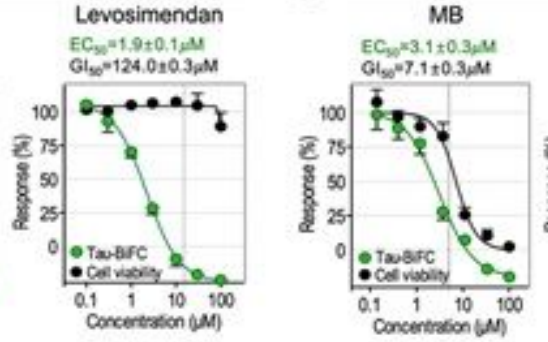

Tau-BiFC ${ }^{\text {ON }}$ by Tauk18 Paoth

+ TauBFCOH

- Tausfor

- FDALitrary

- MB

- UMTM

- Levosimendan

TarAfC by FK

Levosimendan

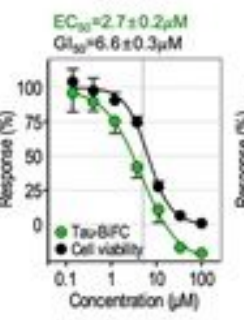

$\mathrm{EC}_{\mathrm{N}}=2.2+0.1, \mathrm{M}$

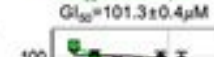

275

25

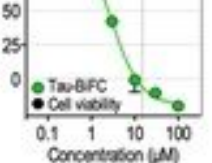

h

Tau-BiFC ${ }^{\mathrm{N}}$ by Tauk18 P3otl.
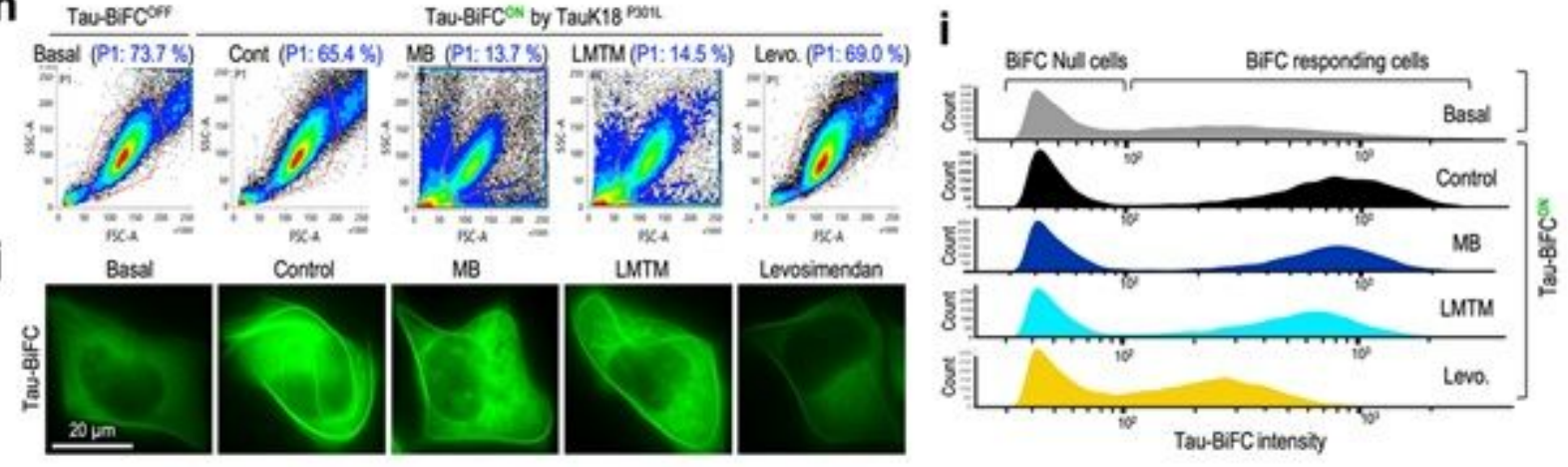

Figure 1

FDA-approved \& Passed Phase I Drug Library screen identifies levosimendan as a new anti-tau agent. a Schematic representation of tau-BiFC screening of FDA-approved \& Passed Phase I Drug Library. For tauBiFC screening, Tau-BiFC cells were treated with each 1,018 library compounds $(10 \mu \mathrm{M})$ in the presence of forskolin (FK). MB and LMTM $(3 \mu \mathrm{M})$ were treated as a positive control. b Scatterplot of 1,018 FDAapproved drug screening. Quadruplicate results of a single drug were plotted with the BiFC intensity on the $\mathrm{Y}$-axis and the number of nuclei on the $\mathrm{X}$-axis. Crosses indicate BiFCOFF-basal, and green dots indicate BiFCON-control. One selective drug, levosimendan (yellow) was plotted with MB (blue) and 
LMTM (cyan). c BiFC fluorescence images of tau-BiFC cells treated with MB, LMTM, or levosimendan in the presence of FK. Nuclei were counterstained with Hoechst and indicated in red color. Scale bar, 100 $\mu \mathrm{m}$. d, e Quantification of tau-BiFC responses (d) and cell viabilities (e). One-way ANOVA with Dunnett's multiple-comparisons test were performed; $* \star p<0.01$, ${ }^{\star \star *} p<0.001$, compared with control. f, g Doseresponse curves of MB, LMTM, and levosimendan with EC50 and GI50 values. In the presence of FK (f) or tauK18P301L (g), each drug was treated to tau-BiFC cells at various concentrations. After 48 hours of treatment, tau-BiFC responses and the number of nuclei were analyzed. A Prism's non-linear regression analysis was used to measure the EC50 and GI50 values. h, i Flow cytometry analysis of tau-BiFC cells co-treated with MB, LMTM $(5 \mu \mathrm{M})$, or levosimendan $(15 \mu \mathrm{M})$ with TauK18P301L. h P1 population indicates mononuclear cells gated with red line based on the level of side scatter (SSC) and forward scatter (FSC), excluding cell debris or dead cells. The percentage of P1 indicates the ratio of healthy, mononuclear cells out of total cells. i BiFC fluorescence histograms of P1 cell populations. j High magnification images of a representative tau-BiFC cell treated with MB, LMTM, and levosimendan. Scale bar, $20 \mu \mathrm{m}$. 


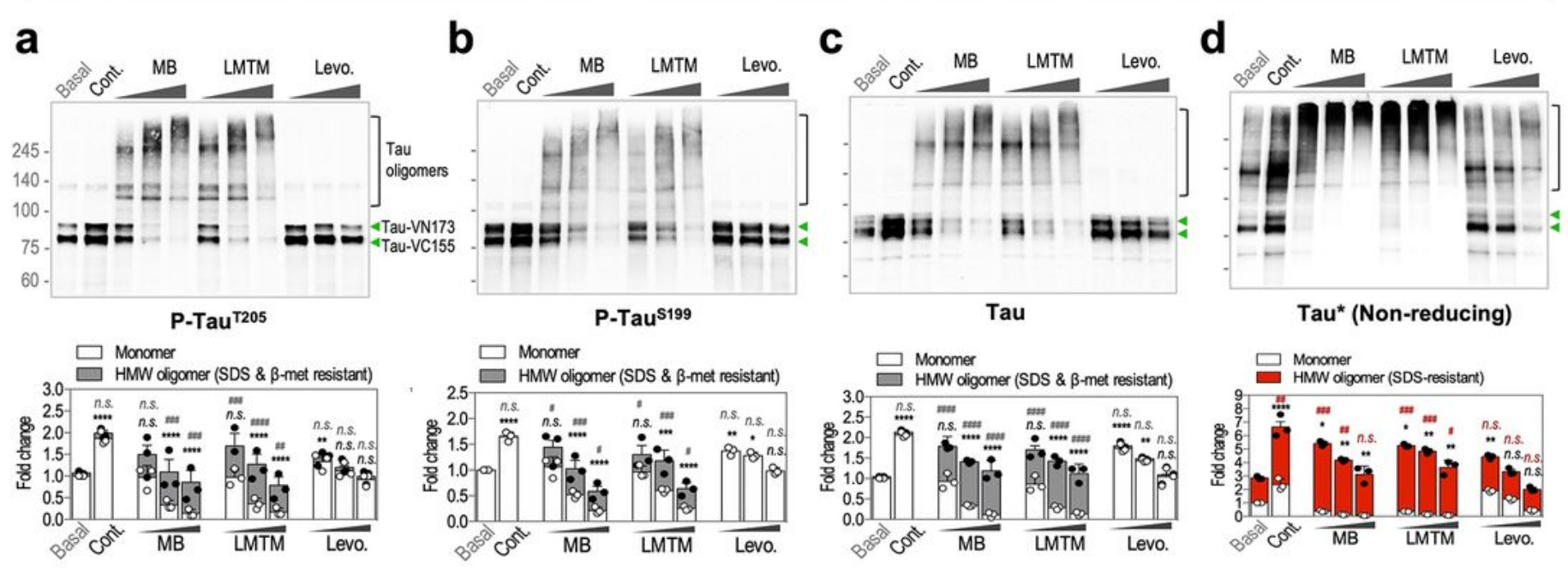

Tau oligomers in primary neurons

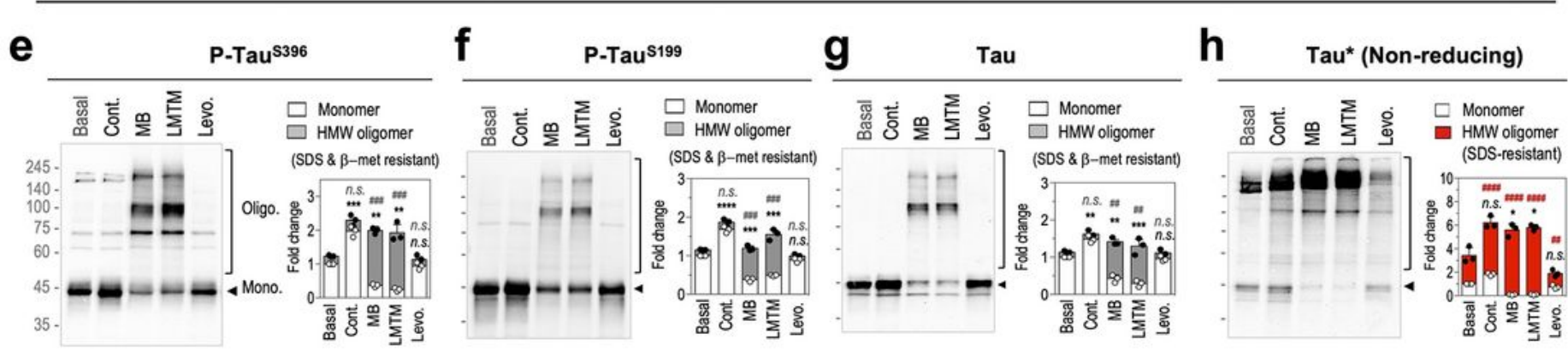

i

Neurotoxic tau oligomers

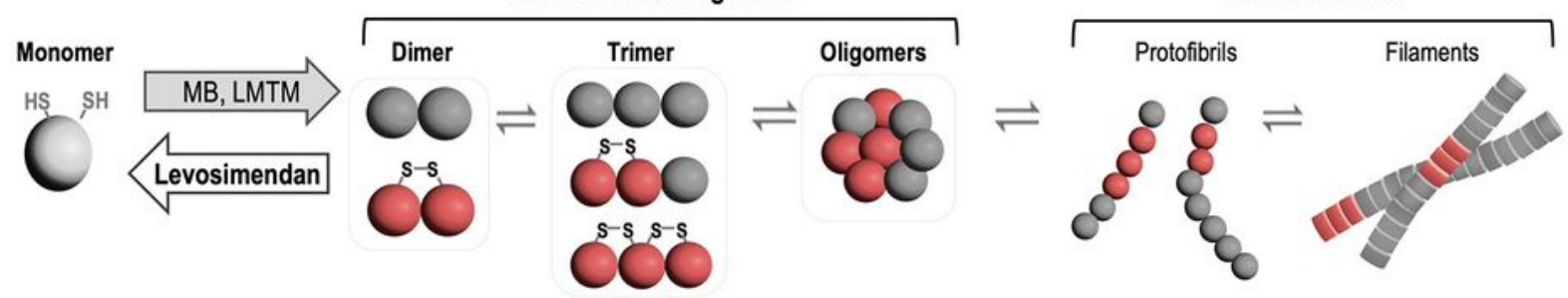

j
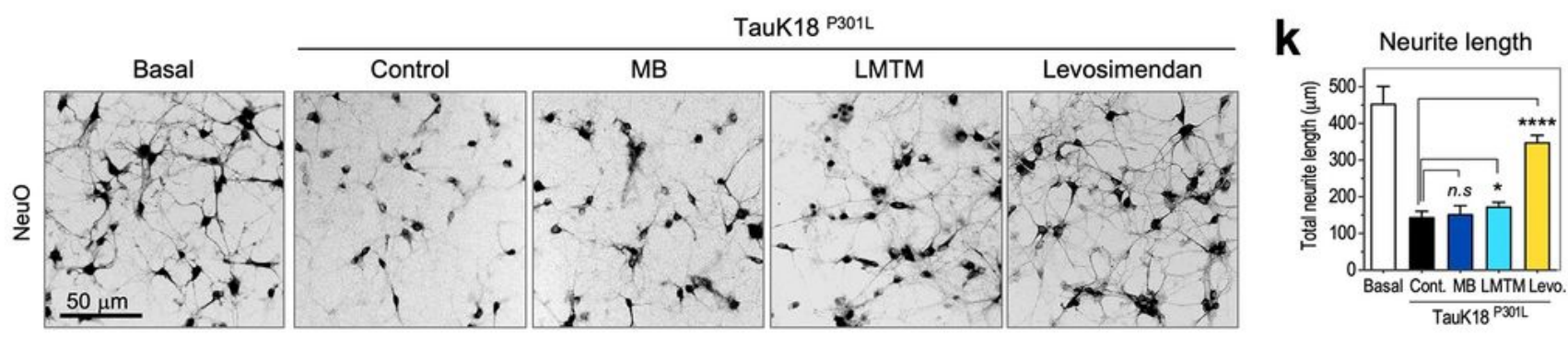

Figure 2

Levosimendan inhibits tau oligomerization in tau-BiFC and primary neuronal cells while MB and LMTM increased tau oligomerization. a-c Immunoblot analysis of total and phospho-tau. For the immunoblot analysis, tau-BiFC cells were treated with $\operatorname{MB}, \operatorname{LMTM}(0.5,1.5,5 \mu \mathrm{M})$, or levosimendan $(5,15,45 \mu \mathrm{M})$ in the presence of tauK18P301L. Green arrows indicate monomer bands of hTau-VN173 and hTau-VC155. Bands above $100 \mathrm{kDa}$ indicates tau oligomers. Relative amounts of monomeric tau (white bars) and 
oligomeric tau (grey bars) were quantified by Image J. $\mathrm{d}$ Immunoblot analysis of total tau on nonreducing condition. Relative amounts of monomeric tau (white bars) and oligomeric tau (red bars) were quantified by Image J. e-g Immunoblot analysis of total and phospho-tau in the lysates of primary neurons. Relative amounts of monomeric and oligomeric tau were quantified by Image J. h Immunoblot analysis of total tau on non-reducing condition. a-h Data represent the mean \pm S.D. of three independent experiments. To indicate significance of the results, Two-way ANOVA with Dunnett's multiplecomparisons test was performed; ${ }^{*} p<0.05,{ }^{*} p<0.01$, ${ }^{* \star} p<<0.001$, ${ }^{\star * * *} p<0.0001$, compared with the level of monomer in basal. \#p $<0.05$, \#\#p $<0.01$, \#\#\#p < 0.001, \#\#\#\#p<0.0001, compared with the level of oligomers in basal. n.s., non-significant. i Schematic diagram of tau aggregation process and the effect of MB, LMTM, or levosimendan on neurotoxic tau oligomerization. j Representative images of primary hippocampal neurons treated with MB, LMTM, or levosimendan. At day 10 in vitro, hippocampal neurons were treated with MB, LMTM $(3 \mu \mathrm{M})$, or Levosimendan $(10 \mu \mathrm{M})$, in the presence of TauK18P301L. After 48hrs, hippocampal neurons were stained with NeuO, a neuron selective probe and imaged using Ti microscope. k Quantification of neurite lengths in NeuO-stained neurons. Scale bar, $50 \mu \mathrm{m}$. The maximal length of neurites was quantified by Image J. Data represent the mean \pm S.D. of three independent experiments. One-way ANOVA with Dunnett's multiple-comparisons test was performed; $* p<0.05, * \star \star * p<0.0001$, compared with control. 


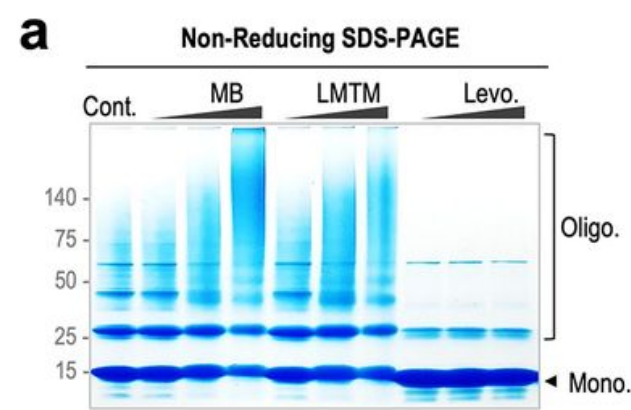

C
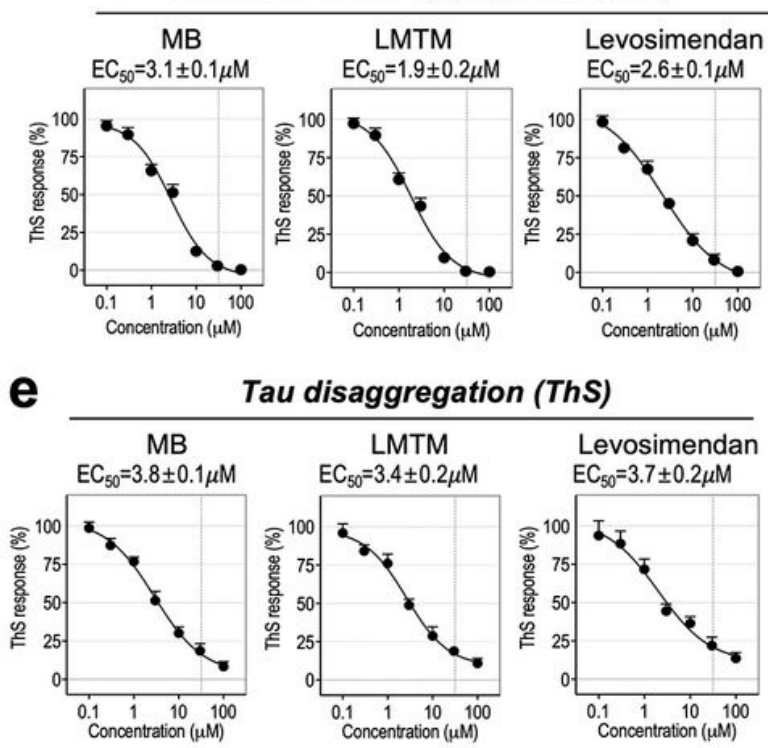

Tau disaggregation (ThS)
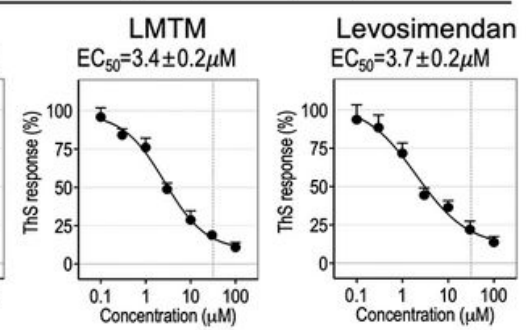

g

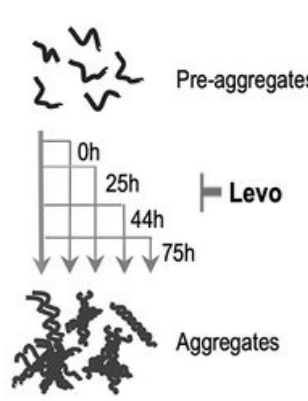

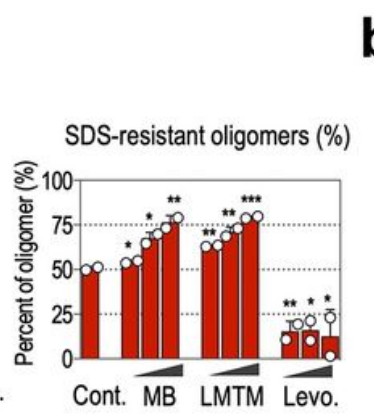

b

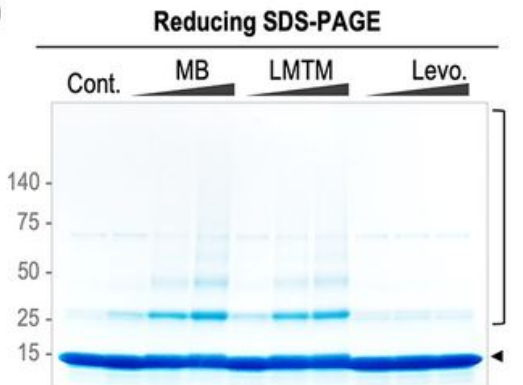

SDS-\& $\beta$-mercaptoethanolresistant oligomers (\%)

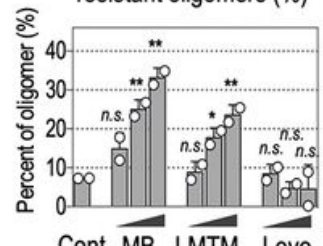

Cont. $\widehat{\text { MB }} \widehat{\text { LMTM }} \widehat{\text { Levo. }}$

d

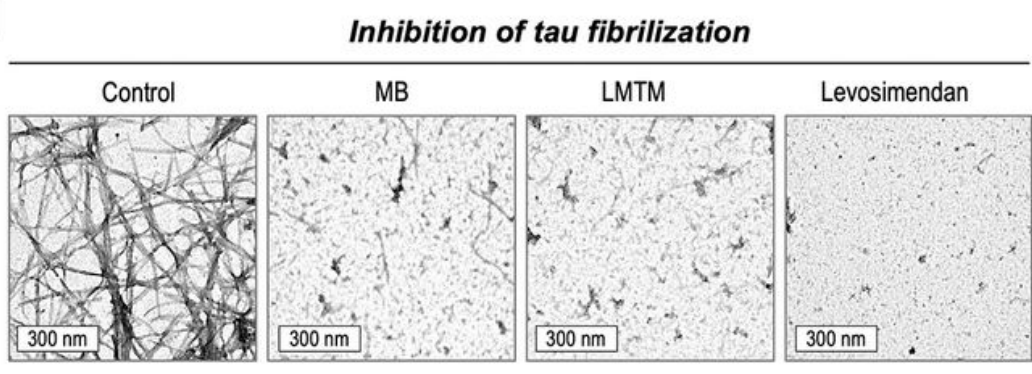

f

Disaggregation of tau fibrils
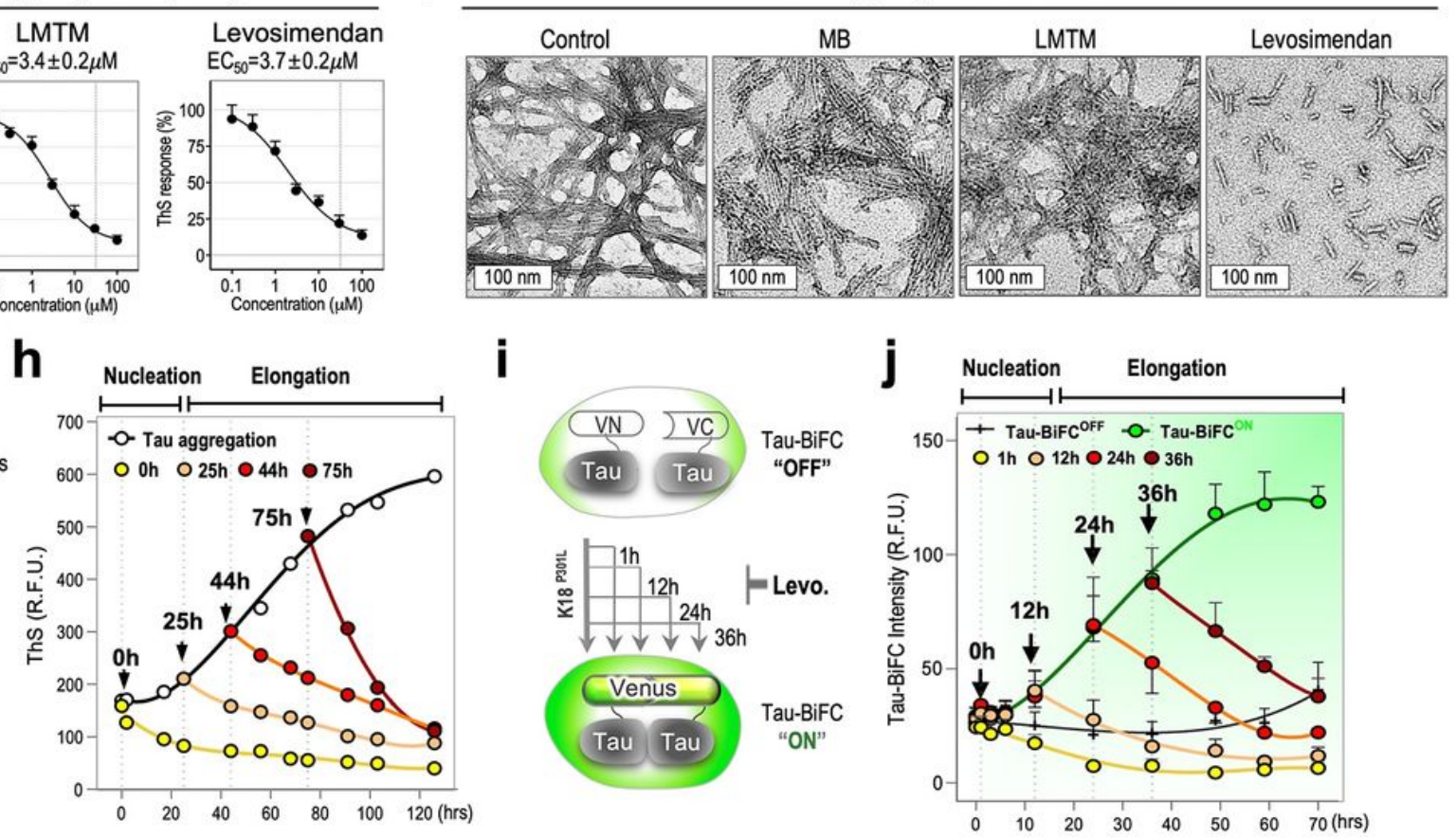

Figure 3

Levosimendan inhibits disulfide-dependent tau oligomerization in vitro. a, b Non-reducing (A) and reducing (B) SDS-PAGE analysis of tau oligomerization in vitro. Tau pre-aggregates were incubated with MB, LMTM, or levosimendan $(10,30,100 \mu \mathrm{M})$ for $5 \mathrm{hrs}$ at RT, and tau proteins were separated on an SDSPAGE gel (4-20\%) under reducing or non-reducing condition. Tau oligomers on SDS-PAGE gels were visualized with Coomassie blue stain. Black arrow indicates tau monomer (14 kDa). Bands above $25 \mathrm{kDa}$ indicate tau oligomers. Relative amounts of tau monomers and oligomers were quantified by Image $\mathrm{J}$. The percentages of SDS-resistant tau oligomers were indicated as red bars (a) and the percentages of SDS- and $\beta$-mercaptoethanol-resistant oligomers were indicated as grey bars (b). Data represent the 
mean \pm S.D. of two independent experiments. n.s., non-significant, ${ }^{\star} p<0.05,{ }^{\star \star} p<0.01$, ${ }^{\star \star \star} p<0.001$, compared with control. c EC50 curves of MB, LMTM, or levosimendan on inhibiting tau aggregation. TauK18 aggregation was induced by heparin and DTT in the presence of each drug for 5 days. The level of $\beta$-sheet aggregation was determined with thioflavin $S(T h S)$. Data represent the mean \pm S.D. of three independent experiments. A Prism's non-linear regression analysis was used to measure the EC50 values. d Representative images of transmission electron microscopy indicating the inhibitory effect of $M B$, LMTM, and levosimendan at $30 \mu \mathrm{M}$. Scale bar, $300 \mathrm{~nm}$. e EC50 curves of MB, LMTM, or levosimendan on disaggregating tau filaments. For disaggregation assay, tau aggregates were incubated with each drug at various concentration for 4 days. The level of $\beta$-sheet aggregation was determined with thioflavin $S$ (ThS). A Prism's non-linear regression analysis was used to measure the EC50 values. Data represent the mean \pm S.D. of three independent experiments. $f$ Representative images of transmission electron microscopy indicating drug' effects on tau disaggregation at $30 \mu \mathrm{M}$. Scale bar, $100 \mathrm{~nm}$. g, h Schematic diagram of the treatment of levosimendan at diverse time points of tau aggregation in vitro $(\mathrm{g})$. Levosimendan $(10 \mu \mathrm{M})$ was treated to an aliquot of the aggregation mixture at $0,25,44$, and $75 \mathrm{hr}$ after heparin treatment. The level of tau aggregation was determined by ThS. (h) ThS-response curves indicating anti-tau aggregation effect of levosimendan in vitro. i, j Schematic diagram of the treatment of levosimendan at various time points of tau-BiFC aggregation (i). Levosimendan was treated to tau-BiFC cells at 1, 12, 24, and $36 \mathrm{hrs}$ after the treatment of tauK18P301L. Tau-BiFC fluorescence images were acquired at various time points and the fluorescence intensities were quantified. (j) Tau-BiFC responses indicating the inhibitory effect of levosimendan on cellular tau aggregation. Data represent the mean \pm S.D. of four repeated measurements. 
a

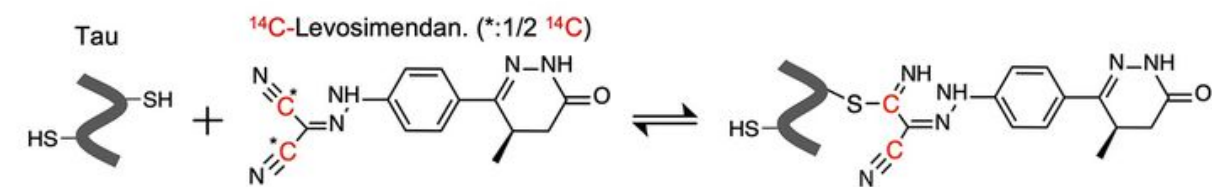

b

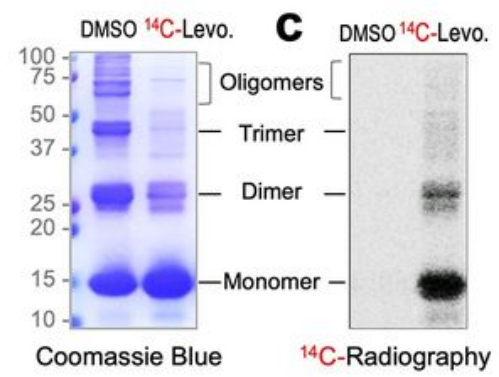

d

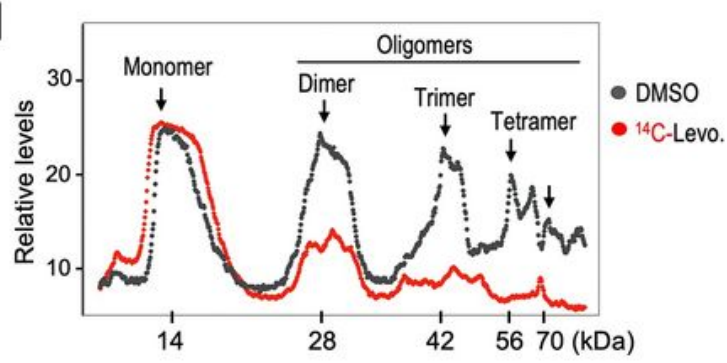

e
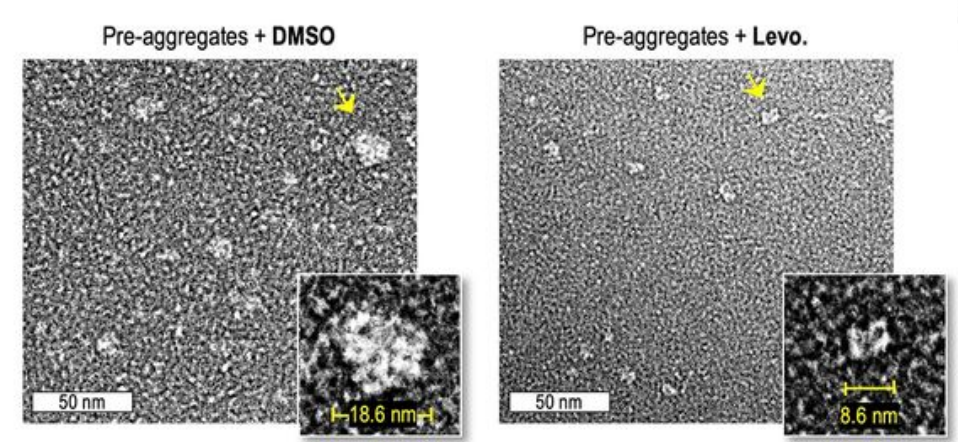

f

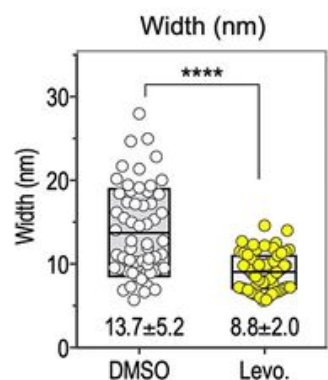

g
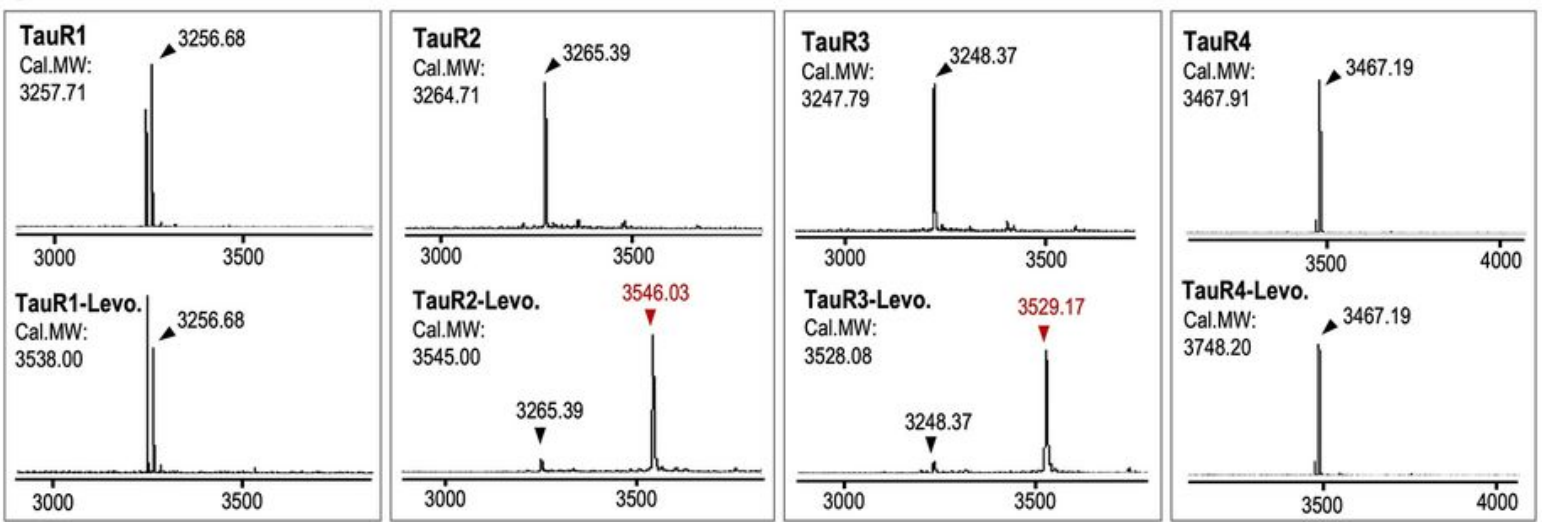

h
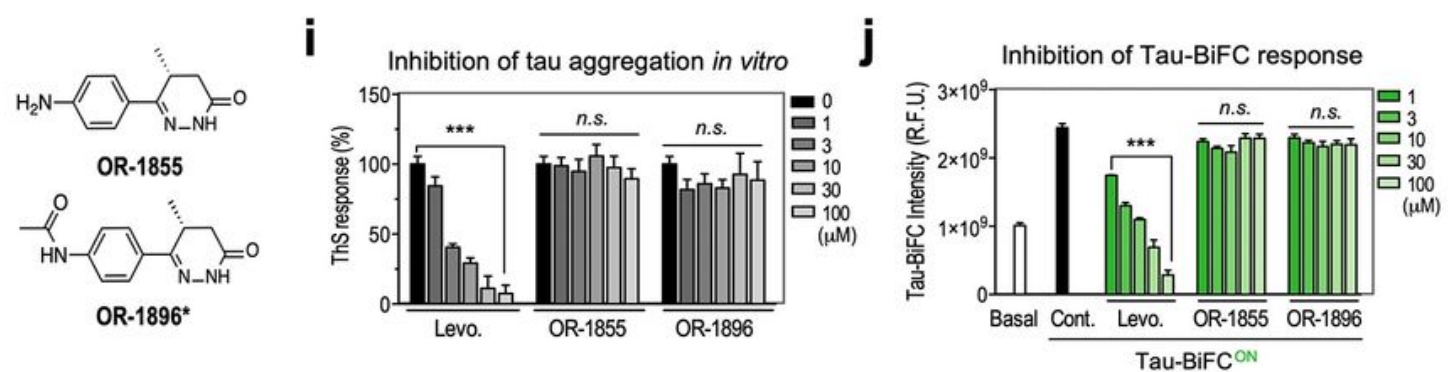

\section{Figure 4}

Levosimendan inhibits disulfide-linked tau oligomerization by capping tau cysteine residues a, c Schematic diagram of a thioimidate bond formation of 14C-levosimendan and tau cysteine (a). TauK18 pre-aggregates were incubated with DMSO control or 14C-levosimendan for $2 \mathrm{hrs}$ at RT. The mixtures were separated on a non-reducing SDS-PAGE gel (15\%) for further analysis. Coomassie blue stain indicates disulfidelinked tau oligomers on the SDS-PAGE gel (b). The SDS-PAGE gel was transferred onto 
a PVDF membrane, and 14C-labelled tau proteins were visualized by autoradiography $14 \mathrm{C}$ autoradiograph (c). $d$ The relative levels of tau monomer and oligomers on the Coomassie Blue stained SDS-PAGE gel. Two independent experiments were performed. e Representative images of transmission electron microscopy indicating disassembly of tau oligomers. A representative tau particle from each group was indicated with a yellow arrow, with its magnified image indicating its width. Scale bar, $50 \mathrm{~nm}$. $f$ Quantification of the widths of tau particles on TEM images. The width of tau particles was measured by Image J. Unpaired t-test was performed; ${ }^{\star \star \star *} \mathrm{p}<0.0001$. g MALDI-TOF analysis of tau repeat domains (R1-R4) in the absence and in the presence of levosimendan. Each repeat domain was incubated with levosimendan for $36 \mathrm{hrs}$ at RT (molar ratio 1:10 of a repeat domain: levosimendan). h Structures of OR1855 and OR-1896, which are not thiol-reactive. i Evaluation of the inhibitory effect of OR-1855 and OR1896 on tau aggregation in vitro by ThS. Data represent the mean \pm S.D. of three repeated experiments. Unpaired t-test was performed; $* \star \star p<0.001$, n.s., non-significant. j Evaluation of the inhibitory effect OR1855 and OR-1896 on tau-BiFC aggregation. Data represent the mean \pm S.D. of three repeated experiments. Unpaired t-test was performed; $* \star * p<0.001$, n.s., non-significant.

a

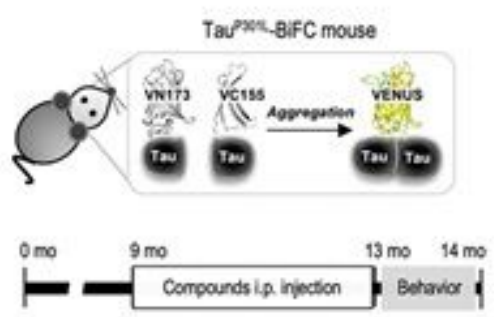

b

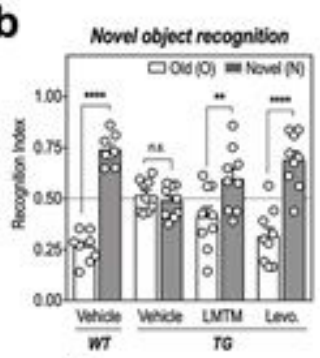

f

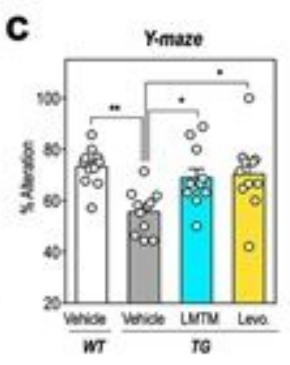

LMTM
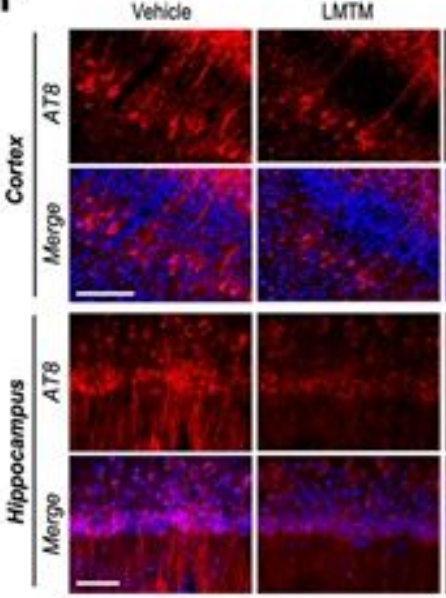

i
Levosimendan
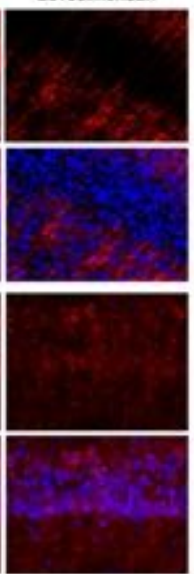

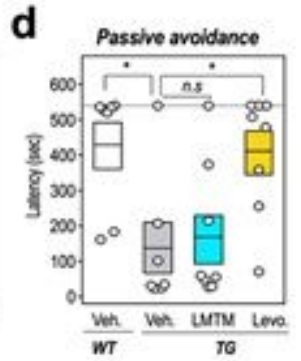

g
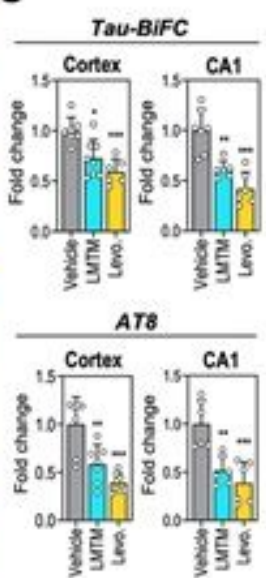

h

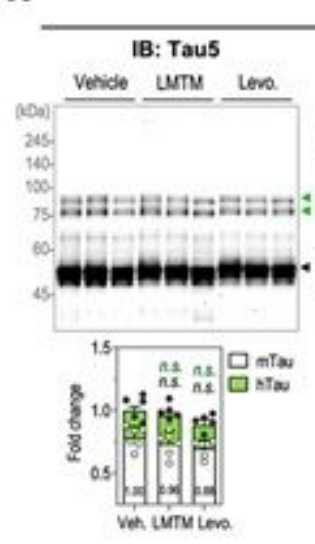

RIPA-soluble tau

IB: p-Tauss

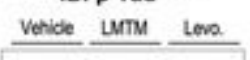

Veride LMTM Leva.

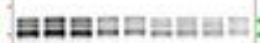
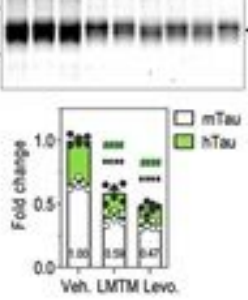

IB: p-Taussos

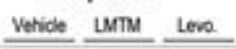

Vehide LMTM Leva.
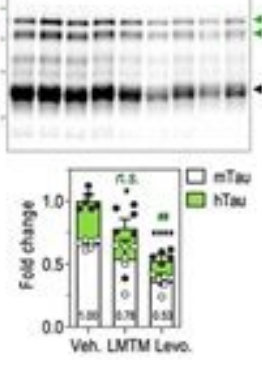

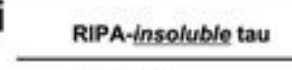

IB: Tau5
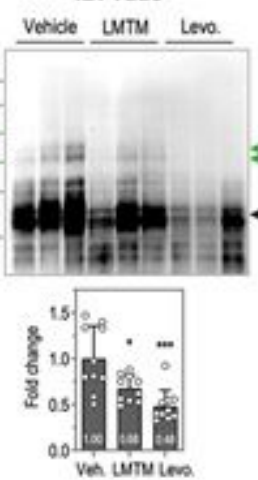

RIPA-soluble tau, Non-reducing

IB: Tau5

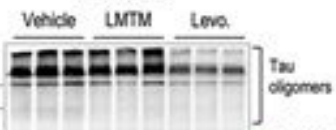

1 RTownerts
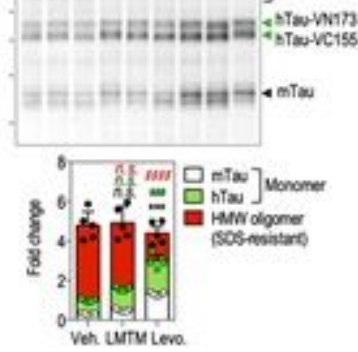


\section{Figure 5}

Levosimendan suppresses tau pathology and cognitive declines in aged TauP301L-BiFC mice. a Diagram of TauP301L-BiFC mouse model and experimental design for administration of LMTM and levosimendan. TauP301L-BiFC mice express human tau mutant (P301L) fused with two non-fluorescent compartments of Venus protein. TauP301L-BiFC mice intraperitoneally received LMTM or levosimendan ( $5 \mathrm{mg} / \mathrm{kg})$ from 9-month-old for 4 months, three times a week $(\mathrm{n}=11)$. b Recognition index (RI) in a novel object recognition test $(n=9)$. RI was defined as the ratio between the times spent exploring the familiar object (Old) or novel object (Novel) divided by total exploratory time. The RI close to 0.5 (dashed line) indicates the mice could not discriminate novel and familiar objects. Two-way ANOVA with Sidak's multiple-comparisons test was performed; $* \star p<0.01, * \star \star \star p<0.0001$. c Percent alteration of $y$-maze test $(n=11)$. A spontaneous alteration was calculated by dividing a number of mouse entering to different arm of the maze in each of 3 consecutive arm entries by the total number of arm entries. One-way ANOVA with Tukey's multiple-comparisons test was performed; ${ }^{\star} p<0.05,{ }^{* \star} p<0.01$. $d$ Latency to enter the dark compartment during the retention test of passive avoidance test $(n=8)$. During training test, each mouse received a foot shock ( $3 \mathrm{~mA}, 2 \mathrm{~s}$ ) upon entry into the dark compartment. Retention test was performed 24 $\mathrm{h}$ after training. One-way ANOVA with Tukey's multiple-comparisons test was performed; $* p<0.05$. e, $f$ Representative images of BiFC fluorescence (e) and AT8-immunofluorescence (f) in cortex and hippocampus of TauP301L-BiFC mouse. Scale bar, $50 \mu \mathrm{m}$. g Quantification of the fluorescence intensities of tau-BiFC and AT8-immunofluorescence $(n=6 \sim 8)$. The mean fluorescence intensities were measured in layer $\mathrm{V}$ of somatosensory cortex and CA1 of hippocampus. The values were normalized to that of vehicletreated group. One-way ANOVA with Tukey's multiple-comparisons test was performed; * $<<0.05$, $* * p<$

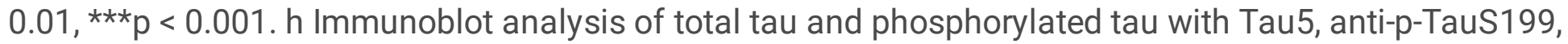
and anti-TauS396 antibodies in RIPA-soluble fractions of TauP301L-BiFC brain lysates $(n=6)$. Green arrows indicate hTau-VN173 and hTau-VC155. Black arrows indicate endogenous murine tau. Relative amounts of human tau (hTau, green bars), murine tau (mTau, white bars) monomers, and total tau (grey bars) were quantified by Image J. i Immunoblot analysis of total tau in RIPA-insoluble fractions. Total tau (grey bars) were quantified by Image $\mathrm{J}$. j Immunoblot analysis of soluble tau oligomers in the brain lysates. To evaluate disulfide-linked tau oligomers, RIPA-soluble brain lysates were separated on a nonreducing SDS-PAGE gel (vehicle, LMTM, levosimendan; $n=10$ ). Relative amounts of hTau (green bars), mTau (white bars) monomers, and oligomers (red bars) were quantified by Image J. h-j To indicate significance of the results, Two-way ANOVA with Tukey's multiple-comparisons test was performed; ${ }^{*} \mathrm{p}<$

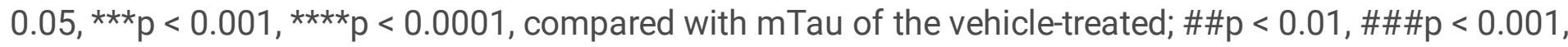
$\# \# \#$ \# $<0.0001$, compared with $\mathrm{hTau}$ of the vehicle-treated; $\S \S \S \S p<0.0001$, compared with oligomer of the vehicle-treated; n.s., nonsignificant.

\section{Supplementary Files}

This is a list of supplementary files associated with this preprint. Click to download. 
- Supplementary4.docx

Page 35/35 